\title{
Gradient-based controllers for timed continuous Petri nets
}

\author{
Dimitri Lefebvre, Edouard Leclercq, Fabrice Druaux \\ Université Le Havre - GREAH, 25 rue P. Lebon, 76063 Le Havre, France \\ IUT Le Havre - GMP, Place R. Schuman, 76600 Le Havre, France
}

\section{Philippe Thomas}

Centre de Recherche en Automatique de Nancy (CRAN-UMR 7039), Université de Lorraine, CNRS, Campus Sciences, B.P. 70239, 54506 Vandœuvre lès Nancy cedex France

\section{Corresponding author: Pr. Dimitri Lefebvre}

Université Le Havre - GREAH, 25 rue P. Lebon, 76063 Le Havre, France

dimitri.lefebvre@univ-lehavre.fr

\begin{abstract}
This paper is about control design for timed continuous Petri nets that are described as piecewise affine systems. In this context, the marking vector is considered as the state space vector, weighted marking of place subsets are defined as the model outputs, and the model inputs correspond to multiplicative control actions that slow down the firing rate of some controllable transitions. Structural and functional sensitivity of the outputs with respect to the inputs are discussed in terms of Petri nets. Then, gradient-based controllers $(G B C)$ are developed in order to adapt the control actions of the controllable transitions according to desired trajectories of the outputs.
\end{abstract}

\section{Introduction}

Petri nets (PN) are useful for the study of discrete event systems (DES) and hybrid dynamical systems (HDS) (Cassandras 1993, Zaytoon et al. 1998) because they combine, in a comprehensive way, intuitive graphical representations and powerful analytic expressions. As a consequence, a lot of results based on PN theory have been established for the control design of DES and HDS. One of the most famous approaches concerns the supervisory control where the system and the controller are considered both as DES (Giua and DiCesare 1994).

Continuous approaches with continuous flow models and continuous PN have been also investigated (David and Alla 2004, Silva and Recalde 2002). The motivation to introduce 
continuous PN are to model the continuous part of HDS and to work out a continuous approximation of DES in order to avoid the complexity associated to the exponential growth of states. Such models have been proved to be suitable to represent the substance level of general semantic models (Zhang and van Luttervelt, 2011) for control and resilience issues. A complete discussion about methods, advantages and limitations of continuous approximations of DES can be found in (Silva and Recalde 2002, 2004) for deterministic systems and in (Lefebvre 2012, Lefebvre and Leclercq 2012, Vasquez and Silva, 2012) for stochastic ones. Flow control design (Lefebvre 1999, Silva and Recalde 2004) have been developed with timed continuous PN (contPN). In particular, controllability and steady states have been characterized (Jimenez et al. 2005, Mahulea et al. 2008, Vasquez et al. 2008) and recently, optimal controls have been investigated. Model predictive control (Giua et al. 2006, Mahulea et al. 2008b, Julvez and Boel 2010), constrained feedback control (Kara et al. 2009, Vazquez and Silva 2009) and linear programming combined with closed loop strategies (ApaydinOzkan et al. 2011) have also been designed. Finally the potential of on/off controllers and of distributed control design has been discussed (Wang et al. 2013). The domains of application are at first manufacturing and traffic systems (Kara et al. 2009, Julvez and Boel 2010, Wang et al. 2013), but computer science and some other domains are also concerned. In the domain of manufacturing systems, Kara et al. (2009) applied constrained feedback control to a simple manufacturing process. Wang et al. $(2011,2013)$ performed a decentralized on/off control to an assembly line with three types of product which are assembled to result in one final product. Apaydin-Ozkan et al. (2011) controlled a flexible manufacturing system (FMS). Tuncel (2012) also studied FMS with colored Petri nets. Chen and Li (2012) investigated the optimal structure of Petri net supervisor for FMS and Chen (2012) exploited colored Petri net to control a RFID based FMS. Lee and Jeong (2011) focused on the control of shared machines by using Petri nets. Some special sessions in recent congresses IEEE SMC 2008, WODES 2012... and special issues of journals have been published on the same subject (Zhou and $\mathrm{Li}, 2010$ ). Other applications of control using different kinds of Petri nets may also be found. In particular, Ross-Leon et al. (2012) exploited contPN models for metabolic systems. Zhang et al. (2011) performed a review on Petri net application for supply chain management. Tolba et al. (2005) investigated continuous PN for the traffic regulation.

In this paper, controllers inspired from artificial intelligence and adaptation algorithms (Widrow and Lehr 1990, Thomas 1997) are proposed for contPN. They are based on sensitivity functions (Lefebvre and Delherm 2003, 2007). For this purpose, contPN are described as piecewise affine models. The system outputs are defined as the marking of 
subsets of places, and the system inputs correspond to control actions that slow down the firing rate of the controllable transitions. The main contributions are to investigate the sensitivity of the PN model from both structural and functional points of view. Such characterizations are used in a systematic way for control issues. Structural sensitivity is helpful to select the transition to control. Then, sensitivity functions are computed and consequently gradient-based controllers (GBC) are proposed that slow down the firing rates of the controllable transitions so that the outputs track reference trajectories. To the best of our knowledge, this class of controllers has not been yet investigated. The advantage of the GBC is the systematic numerical implementation for multi-inputs and multi-outputs (MIMO) models of DES and HDS. The usual discussion about structural properties of PN (join-free, choice free, consistency, conservativeness, and so on) is replaced by the sensitivity analysis that has an intuitive meaning for control issues.

The paper is divided into 5 sections. The section 2 is about PN and contPN. The section 3 concerns the structural analysis that provides useful results concerning output structural sensitivity. The section 4 is about the design of GBC. Various examples of contPN are proposed in section 5 in order to discuss the proposed results and to compare GBC with proportional - integral, on/off controllers and model predictive controller.

\section{Petri nets}

A marked Petri net (PN) with $n$ places and $q$ transitions is defined as $\left\langle\boldsymbol{P}, \boldsymbol{T}, W_{P R}, W_{P O}, M_{I}\right\rangle$ where $\boldsymbol{P}=\left\{P_{i}\right\}_{i=1, \ldots, n}$ is a set of places, $\boldsymbol{T}=\left\{T_{j}\right\}_{j=1, \ldots, q}$ is a set of transitions, such that $\boldsymbol{P} \cap \boldsymbol{T}=\varnothing$. $W_{P R}=\left(w^{P R}{ }_{i j}\right) \in\left(\mathbf{Z}^{+}\right)^{n \times q}$ is the pre-incidence matrix and $W_{P O}=\left(w^{P O}{ }_{i j}\right) \in\left(\mathbf{Z}^{+}\right)^{n \times q}$ is the postincidence matrix where $\mathbf{Z}^{+}$is defined as the set of non-negative integer numbers. The PN incidence matrix $W$ is defined as $W=W_{P O}-W_{P R} \in\left(\mathbf{Z}^{+}\right)^{n \times q}$. Let us also define $M=\left(m_{i}\right) \in$ $\left(\mathbf{Z}^{+}\right)^{n}$ as the marking vector and $M_{I} \in\left(\mathbf{Z}^{+}\right)^{n}$ as the initial marking vector. ${ }^{\boldsymbol{O}} \boldsymbol{T}_{\boldsymbol{j}}$ (resp $\boldsymbol{T}_{\boldsymbol{j}}{ }^{\circ}$ ) stands for the pre-set (resp. post-set) places of $T_{j}$. When two transitions $T_{j}$ and $T_{j}$, have a common place in the pre-set, the PN presents a structural conflict. The conflict is an effective one if there are not enough tokens in the common place to fire both transitions.

\subsection{Timed continuous Petri nets}

Timed continuous PN under infinite server semantics (contPN) provide a continuous approximation of DES behaviour (David and Alla 2004). A marked contPN is defined as < PN, $X_{\max }>$ where PN is a marked Petri net and $X_{\max }=\operatorname{diag}\left(x_{\max j}\right) \in\left(\mathbf{R}^{+}\right)^{q}$ is the matrix of maximal transition firing rates with $\mathbf{R}^{+}$the set of non-negative real numbers. The marking 
$m_{i}(t)$ of each place $P_{i}, i=1, \ldots, n$, at time $t$ has a non-negative real value and each transition firing is a continuous flow in contPN. Let us define $X(t)=\left(x_{j}(t)\right) \in\left(\mathbf{R}^{+}\right)^{q}$ as the firing rate (i.e. flow) vector at time $t$ which continuously depends on the marking of the places according to equations (1) and (2):

$x_{j}(t)=x_{\max j} \cdot \mu_{j}(t)$

with:

$\mu_{j}(t)=\min _{P_{i} \in \mathcal{O}^{\circ} T_{j}}\left(\frac{m_{i}(t)}{w_{i j}^{P R}}\right)$

The marking variation is given by the differential system (3):

$$
\begin{aligned}
& \frac{d M(t)}{d t}=W \cdot X(t) \\
& M(0)=M_{I}
\end{aligned}
$$

Due to the function « $\min »$ in the expression of the enabling degree (2), contPN are not linear but piecewise linear systems (Lefebvre et al. 2003, Silva and Recalde 2004). Let us introduce the critical place(s) for transition $T_{j}$ at time $t$ as the place(s) $P_{k}$ such that $k$ correspond(s) to the value(s) of the index $i$ for which the quantity of tokens $m_{i}(t) / w^{P R}{ }_{i j}$ is minimal for all $P_{i} \in{ }^{\circ} \boldsymbol{T}_{j}$. Let us notice that a transition may have several critical places. For each marking $M(t)$ a single critical place is selected with the $q$ functions $f_{j}$ :

$$
\begin{aligned}
\forall T_{j} \in \boldsymbol{T}, \quad f_{j}: & \left(\mathbf{R}^{+}\right)^{n} \rightarrow\{1, \ldots, n\} \\
M(t) & \rightarrow f_{j}(M(t))=\min \left\{i \text { such that } m_{i}(t)=\mu_{j}(t) . w_{i j}\right\}
\end{aligned}
$$

Function "min" is used in (4) to select a unique place (the one with smallest index) in the set of critical places. This place is noted $m_{f j}(t)$ in the following. According to the functions $f_{j}$, the set of reachable markings is partitioned into a finite number $K$ of regions $\mathbf{A}_{\boldsymbol{\varphi}}$ with $K \leq \Pi\left\{{ }^{\circ} \boldsymbol{T}_{j} \mid\right.$, $j=1, \ldots, q$. Each region $\mathbf{A}_{\varphi}$ is characterised by a constraint matrix $A_{\varphi}=\left(a^{\varphi}{ }_{i j}\right) \in\left(\mathbf{R}^{+}\right)^{q \times n}$ with, where $a_{j i}^{\varphi}=1 / w^{P R}{ }_{i j}$ if $i=f_{j}(M(t))$ and $a_{j i}^{\varphi}=0$ otherwise (Lefebvre 2011). As a consequence, contPN are piecewise-affine hybrid systems with a linear expression in each region $\mathbf{A}_{\varphi}$ :

$\forall M(t) \in \mathbf{A}_{\varphi}, d M(t) / d t=W \cdot X_{\max } \cdot A_{\varphi} M(t)$ 
From a behavioural point of view, several phases occur in the marking trajectories. Each phase is active in a particular region $\mathbf{A}_{\varphi}$ or PN configuration (Mahulea et al. 2008).

\subsection{An introductive example}

ContPN can be extended with non linear firing speeds and enlarged with discrete places and transitions to approximate DES or HDS (Balduzzi et al. 2000, Zaytoon et al. 1998) as illustrated with the example in figure 1, modelled with the hybrid PN in figure 2.

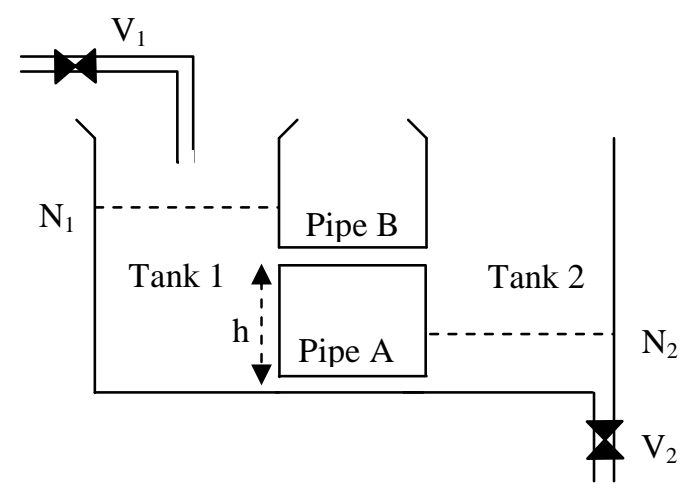

Figure 1: Two-tank system (system A)

[Insert figure 1 here]

The places $P_{1}$ and $P_{2}$ are continuous and the markings $m_{1}$ and $m_{2}$ stand respectively for the height of liquid in both tanks 1 and $2\left(m_{1}(t) \geq m_{2}(t)\right.$ for all $\left.t \geq 0\right)$ according to (6):

$S_{1} \cdot \dot{m}_{1}(t)=x_{1}(t)-x_{2}(t)-x_{3}(t)$
$S_{2} \cdot \dot{m}_{2}(t)=x_{2}(t)+x_{3}(t)-x_{4}(t)$

where $S_{1}$ and $S_{2}$ stand for the sections of tanks 1 and 2 (for simulations $S_{1}=S_{2}=0.0154 \mathrm{~m}^{2}$ ). The initial marking vector of the continuous part of the model is $M_{I}=(0,0)^{\mathrm{T}}$. The transitions $T_{1}$ to $T_{4}$ are continuous and their firings represent the input flow $\left(x_{1}\right)$, the output flow $\left(x_{4}\right)$ and the flows through the pipes $\mathrm{A}\left(x_{2}\right)$ and $\mathrm{B}\left(x_{3}\right)$ according to $(7)$ :

$$
\begin{aligned}
& x_{1}(t)=D \\
& x_{2}(t)=\alpha_{2} \cdot \sqrt{m_{1}(t)-m_{2}(t)} \\
& x_{3}(t)=\alpha_{3} \cdot \sqrt{\sup \left(m_{1}(t), h\right)-\sup \left(m_{2}(t), h\right)} \\
& x_{4}(t)=\alpha_{4} \cdot \sqrt{m_{2}(t)}
\end{aligned}
$$


where $D, \alpha_{2}, \alpha_{3}, \alpha_{4}$ and $h$ are related to the system specifications (for simulations $\alpha_{2}=\alpha_{3}=\alpha_{4}$ $=1.6 \cdot 10^{-4} \mathrm{~m}^{5 / 2} \cdot \mathrm{s}^{-1}, D=1.10^{-4} \mathrm{~m}^{3} \cdot \mathrm{s}^{-1}$ and $\left.h=0.5 \mathrm{~m}\right)$.

The discrete part of the PN (i.e. places $P_{3}$ and $P_{4}$ and transitions $T_{5}$ and $T_{6}$ ) stands for the controller. A token in $P_{3}$ means that valve $V_{1}$ is open and $V_{2}$ is closed. On the contrary, a token in $P_{4}$ means that valve $V_{2}$ is open and $V_{1}$ is closed. The arcs from $P_{1}$ to $T_{5}$ and from $P_{2}$ to $T_{6}$ are test arcs (the value of the places $P_{1}$ and $P_{2}$ is not changed by firing the transitions $T_{5}$ and $T_{6}$ ). The goal of the controller is to open $V_{1}$ and close $V_{2}$ when $m_{2}(t)<y^{\text {des }}{ }_{2}(t)$ and to open $V_{2}$ and close $V_{l}$ when $m_{l}(t)>y^{\text {des }}{ }_{l}(t)$, where $y^{\text {des }}{ }_{1}(t)$ and $y^{\text {des }}{ }_{2}(t)$ are the desired trajectories for $m_{1}(t)$ and $m_{2}(t)$ that satisfy $y^{\text {des }}(t) \geq y^{\text {des }}{ }_{2}(t)$. When $y^{\text {des }}{ }_{1}(t)$ and $y^{\text {des }}{ }_{2}(t)$ have constant values, this discrete control design results in a cyclic behaviour. In section 4 , we propose to replace the discrete controller (figure 16) with a GBC (figure 15) useful to reach desired levels or to track reference trajectories.

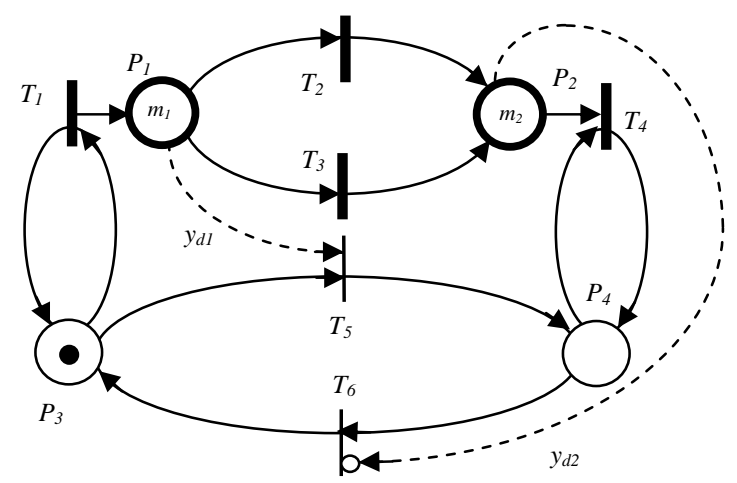

Figure 2: Hybrid PN of the two-tank system

[Insert figure 2 here]

\subsection{Controlled timed continuous Petri nets}

For control issues, the set of transitions $\boldsymbol{T}$ is divided into 2 disjoint subsets $\boldsymbol{T}_{C}$, and $\boldsymbol{T}_{N C}$ such that $\boldsymbol{T}=\boldsymbol{T}_{\boldsymbol{C}} \cup \boldsymbol{T}_{N C} . \boldsymbol{T}_{\boldsymbol{C}}$ is the subset of the $d$ controllable transitions, and $\boldsymbol{T}_{N C}$ is the uncontrollable transitions subset with cardinal $q-d$. A particular case is given by $\boldsymbol{T}_{\boldsymbol{C}}=\boldsymbol{T}$ and $\boldsymbol{T}_{N C}=\varnothing$, but in many cases, not all transitions are controllable. For instance, the transitions $T_{2}$ and $T_{3}$ in figure 2 correspond to the flows through the pipes $\mathrm{A}$ and $\mathrm{B}$ that are not controllable in the sense that these pipes have no valve.

Control actions are introduced for contPN according to a reduction in the flow through the transitions (Jimenez et al. 2005). Such control actions can be interpreted as slowing down the server activities in the considered systems. Multiplicative and additive control actions have 
been introduced and discussed. Both approaches are quite equivalent (Silva and Recalde 2004) and in this paper multiplicative control actions are considered.

Let us introduce $U(t)=\left(u_{j}(t)\right) \in\left(\mathbf{R}^{+}\right)^{q}$ with $0 \leq u_{j}(t) \leq 1$ for $T \in \boldsymbol{T}_{\boldsymbol{C}}$ and $u_{j}(t)=1$ for $T \in \boldsymbol{T}_{N C}$ as the contPN input vector at time $t$. The proposed control actions slow down the flow through the controllable transitions and do not change the firing rates of uncontrollable transitions. The controlled firing speed vector $X_{C}(t)=\left(x_{C j}(t)\right)_{j=1, \ldots, q} \in\left(\mathbf{R}^{+}\right)^{q}$ is defined with (8):

$x_{C j}(t)=x_{\max } \cdot u_{j}(t) \cdot \mu_{j}(t)$

The output vector $Y(t)=Q \cdot M(t) \in\left(\mathbf{R}^{+}\right)^{e}$ is composed of a selection of $e$ subsets of places whose global marking is measured. For this purpose, let us define $Q=\left(q_{\alpha i}\right) \in\left(\mathbf{R}^{+}\right)^{e \times n}$ as an observation matrix (i.e. a projector). Each row of $Q$ corresponds to a weighted sum of the PN places marking. As a consequence, marking measurement concerns not only individual places but also groups of places. The goal of the controller is to drive $Y(t)$ according to some reference trajectories in the output space. The marking variation of controlled contPN is rewritten with (9):

$$
\begin{aligned}
& \frac{d M(t)}{d t}=W \cdot X_{C}(t) \\
& Y(t)=Q \cdot M(t)
\end{aligned}
$$

In each region $\mathbf{A}_{\varphi}$ and during each phase, a constant relationship exists between the components of vectors $X_{C}(t)$ and $M(t)$. This relation can be expressed in scalar form with the functions $f_{j}$ :

$x_{C j}(t)=\left(x_{\max j} / w^{P R}{ }_{f j} j\right) \cdot u_{j}(t) \cdot m_{f j}(t), j=1, \ldots, q$

or in vectorial form with the constraint matrices $A_{\varphi}$ :

$\forall M(t) \in \mathbf{A}_{\varphi,} X_{C}(t)=X_{\max } \cdot \operatorname{diag}(U(t)) \cdot A_{\varphi} M(t)$

Equation (9) can be rewritten in scalar form:

$$
\begin{aligned}
& \frac{d m_{i}(t)}{d t}=\sum_{j=1}^{q}\left(\frac{w_{i j} \cdot x_{\max j}}{w^{P R}{ }_{f j} j}\right) \cdot u_{j}(t) \cdot m_{f_{j}}(t), \quad i=1, \ldots, n \\
& y_{\alpha}(t)=\sum_{i=1}^{n} q_{\alpha i} \cdot m_{i}(t), \quad \alpha=1, \ldots, e
\end{aligned}
$$

or in vectorial form, for all $M(t) \in \mathbf{A}_{\varphi}$ : 


$$
\begin{aligned}
& \frac{d M(t)}{d t}=W \cdot X_{\text {max }} \cdot \operatorname{diag}(U(t)) \cdot A_{\varphi} \cdot M(t) \\
& Y(t)=Q \cdot M(t)
\end{aligned}
$$

The design of GBC for contPN includes structural and functional aspects:

- The structural analysis is necessary to determine which inputs act on a given output. It is useful to select the controllable transitions. In section $3, W$-sensitivity is introduced and structural analysis is discussed.

- The functional analysis consists in adapting the usual gradient algorithm in order to drive the contPN outputs near the desired marking. In section 4, sensitivity functions are defined and worked out to design GBC.

\section{Structural analysis}

The structural analysis provides qualitative results useful to study the controllability of PN models (David and Alla 2004).

\subsection{W-sensitivity}

This section concerns the structural sensitivity, referred as $W$-sensitivity in the following, of the outputs with respect to (wrt) the variations of the PN inputs. The $W$-sensitivity depends only on the structure of the PN models and provides conditions that are required for the control design of PN and that will be used in section 4. This study is based on the $W$ sensitivity of the places and transitions wrt the PN firing conditions (Lefebvre and Delherm 2003, 2007).

Definition 3.1: The node $N$ (i.e. transition $T_{j} \in \boldsymbol{T}$ or place $P_{i} \in \boldsymbol{P}$ ) is $W$-sensitive wrt the transition $T_{\gamma} \in \boldsymbol{T}$ if the firings of $T_{\gamma}$ could influence the variable attached to $N$ (i.e. the marking $m_{i}$ of place $P_{i}$ or the firing $x_{j}$ of transition $T_{j}$ ). In this case there exists a causality relationship from transition $T_{\gamma}$ to node $N$.

The $W$-sensitivity of the outputs wrt the variations of the PN inputs is defined as a consequence. 
Definition 3.2: For any controllable transitions $T_{\gamma} \in \boldsymbol{T}_{\boldsymbol{C}}$ and any output $y_{\alpha}$, the ouput $y_{\alpha}$ is $W$ sensitive wrt the input $u_{\gamma}$ if a variation of $u_{\gamma}$ could influence at least the marking of one place in the subset of places corresponding to $y_{\alpha}$.

If $y_{\alpha}$ is $W$-sensitive wrt the input $u_{\gamma}$ there exists a causality relationship from input $u_{\gamma}$ to output $y_{0}$. The causality relationships can be worked out with the pre and post incidence matrices, according to the theorem 3.1 .

Theorem 3.1: The output $y_{\alpha}$ is $W$-sensitive wrt the input $u_{\gamma}$ if and only if there exists an integer $r \in[0, \min (n, q)]$ such that equation (14) holds:

$C_{\alpha}^{T} \cdot Q \cdot\left(\left(W_{P R}+W_{P O}\right) \cdot\left(W_{P R}\right)^{T}\right)^{r} \cdot\left(W_{P R}+W_{P O}\right) \cdot B_{\gamma} \neq 0$

with $B_{\gamma}=\left(b_{j}^{\gamma}\right) \in\{0,1\}^{q}$ such that $b_{j}^{\gamma}=0$ if $\gamma \neq j$ and $b_{\gamma}^{\gamma}=1, C_{\alpha}=\left(c_{j}^{\alpha}\right) \in\{0,1\}^{e}$ such that $c^{\alpha}{ }_{j}=0$ if $\alpha \neq j$ and $c^{\alpha}{ }_{\alpha}=1$.

Proof: Let us first notice that a change of the firing conditions of transition $T_{\gamma}$ yields a deviation of the places marking near $T_{\gamma}$ (i.e. ${ }^{\circ} \boldsymbol{T}_{\gamma} \cup \boldsymbol{T}_{\gamma}{ }^{\circ}$ ) from its true value. This deviation is likely to change the firing of the downstream transitions $\left(\left({ }^{\circ} \boldsymbol{T}_{\gamma} \cup \boldsymbol{T}_{\gamma}^{\circ}\right)^{\circ}\right)$. In fact, the initial perturbation could propagate in the PN according to the following rules (figure 3).

1) A change of the firing conditions of any transition $T_{\gamma}$ yields a deviation of the $T_{\gamma}$ - input and $T_{\gamma}$ - output places marking (i.e. ${ }^{\circ} \boldsymbol{T}_{\gamma} \cup \boldsymbol{T}_{\gamma}{ }^{\circ}$ ) from its true value. The change could also influence the firing conditions of any other transition $T_{j}$ if the $T_{j}$ - input places (i.e. ${ }^{\circ} \boldsymbol{T}_{j}$ ) marking is modified.

2) A deviation of the marking of any place $P_{i}$ influences the firing conditions of the $P_{i}-$ downstream transitions (i.e. $\boldsymbol{P}_{i}^{\circ}$ ) and the marking of any place $P_{\alpha}$ has a structural sensitivity wrt $P_{\alpha}$ - upstream and $P_{\alpha}$ - downstream transitions (i.e. ${ }^{\circ} \boldsymbol{P}_{\boldsymbol{\alpha}} \cup \boldsymbol{P}_{\boldsymbol{\alpha}}{ }^{\circ}$ ).

The characterisation of the neighbourhood in PN results from the algebraic properties of the post and pre incidence matrices:

- The position of the non-zero entries of the $j^{\text {th }}$ column in $W_{P R}$ (resp. in $W_{P O}$ ) corresponds to the $T_{j}$ - input places (resp. $T_{j}$ - output places).

- The position of the non-zero entries of the $i^{\text {th }}$ row in $W_{P R}$ (resp. in $W_{P O}$ ) corresponds to the $P_{i}$ - downstream transitions (resp. $P_{i}$ - upstream transitions). 
- The position of the non-zero entries of the $j^{\text {th }}$ column in $W_{P R}+W_{P O}$ (resp. the $i^{\text {th }}$ row in $\left.W_{P R}+W_{P O}\right)$ corresponds to the places (resp. transitions) next to $T_{j}$ (resp. $P_{i}$ ).

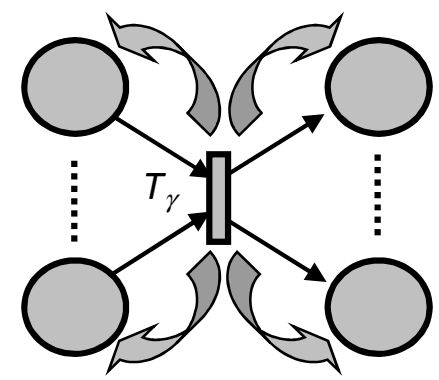

a) Sensitivity of the places near $T_{\gamma}$ wrt the firing conditions of $T_{\gamma}$

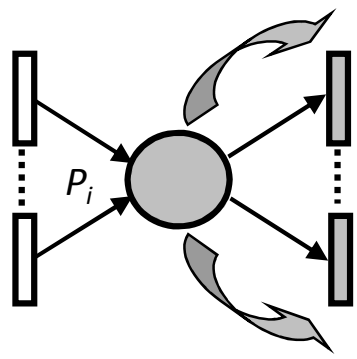

c) Sensitivity of the transitions near $P_{i}$ wrt the marking of $P_{i}$

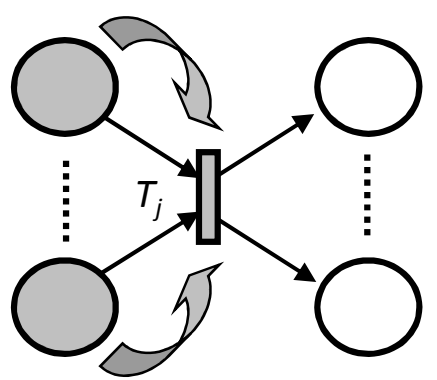

b) Sensitivity of $T_{j}$ wrt the marking of the places near $T_{j}$

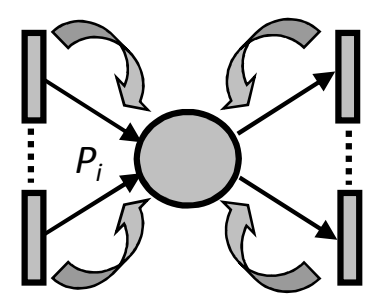

d) Sensitivity of $P_{\alpha}$ wrt the firing conditions of the transitions near $P_{\alpha}$

Figure 3: Propagation of the perturbation near a given transition or place

[Insert figure 3 here]

The set of places that are structurally sensitive wrt the firing conditions of $T_{\gamma} \in \boldsymbol{T}_{\boldsymbol{C}}$ is worked out with a recursive algorithm. The position of the non-zero entries of the $\gamma^{\text {th }}$ column in $W_{P R}+$ $W_{P O}$ corresponds to the places near $T_{\gamma}$. The position of the non-zero entries of the $\gamma^{\text {th }}$ column in $\left(W_{P R}\right)^{\mathrm{T}} \cdot\left(W_{P R}+W_{P O}\right)$ corresponds to the downstream transitions near the places near $T_{\gamma}$ The position of the non-zero entries of the $\gamma^{\text {th }}$ column in $\left(W_{P R}+W_{P O}\right) \cdot\left(W_{P R}\right)^{\mathrm{T}} \cdot\left(W_{P R}+W_{P O}\right)$ corresponds to the places near the downstream transitions near the places near $T_{\gamma}$ Matrices $\left(\left(W_{P R}+W_{P O}\right) \cdot\left(W_{P R}\right)^{\mathrm{T}}\right)^{2} \cdot\left(W_{P R}+W_{P O}\right) \ldots\left(\left(W_{P R}+W_{P O}\right) \cdot\left(W_{P R}\right)^{\mathrm{T}}\right)^{r} \cdot\left(W_{P R}+W_{P O}\right)$ are successively computed. When the PN has $n$ places and $q$ transitions, the structural sensitivity analysis of the places and transitions is completed in a finite number $r$ of steps no larger than $\min (n, q)$.

The output $W$ - sensitivity matrix $\Sigma_{W}$ is defined consequently. 
Definition 3.3: The output $W$-sensitivity matrix is defined as $\Sigma_{W}=\left(\sigma_{W \alpha \gamma}\right) \in\{[0, \min (n, q)] \cup$ $\infty\}^{e \times q}$ with $\sigma_{W \alpha \gamma}$ given by equation (15):

$$
\sigma w_{\alpha \gamma}=\min _{r \in[0, \min (n, q)] \cup \infty}\left\{C_{\alpha}^{T} \cdot Q \cdot\left(\left(W_{P R}+W_{P O}\right) \cdot\left(W_{P R}\right)^{T}\right)^{r} \cdot\left(W_{P R}+W_{P O}\right) \cdot B_{\gamma} \neq 0\right\}
$$

$\sigma_{W \alpha \gamma}$ equals either infinity if $y_{\alpha}$ is not $W$-sensitive wrt input $u_{\gamma}$ or the number of intermediate places in the shortest causality relationship from $u_{\gamma}$ to the subset of places corresponding to $y_{\alpha}$ if $y_{\alpha}$ is $W$-sensitive wrt input $u_{\gamma}$ (Lefebvre et al. 2003, Lefebvre and Delherm 2007). In this last case, $\sigma_{W \alpha \gamma}$ is named the $W$-sensitivity rank of $y_{\alpha}$ wrt $u_{\gamma}$. The output $W$ - sensitivity matrix provides immediate results about the causality relationships in $\mathrm{PN}$, as explained in theorem 3.2:

Theorem 3.2: The set of outputs (resp. rank - $r$ outputs) that are $W$ - sensitive wrt input $u_{\gamma}$ is given by the position of the finite entries (resp. entries with value $r$ ) of the $\gamma^{\text {th }}$ column in matrix $\Sigma_{W}$.

The set of inputs (resp. rank - $r$ inputs) whose firing conditions are likely to influence the output $y_{\alpha}$ is given by the position of the finite entries (resp. entries with value $r$ ) of the $\alpha^{\text {th }}$ row in matrix $\Sigma_{W}$.

Proof: the proof of theorem 3.2 is obvious and results from definition 3.2 and theorem 3.1 .

\subsection{Examples}

In order to illustrate the $W$ - sensitivity analysis, the following examples are proposed. The contPN B with the marking vector $M(t)=\left(m{ }^{\prime}{ }_{0}(t), m^{\prime}{ }_{1}(t), m{ }_{2}(t), m_{1}(t), m_{2}(t), m^{\prime}{ }_{1}(t), m^{\prime}{ }_{2}(t)\right)^{\mathrm{T}}$ shown in figure 4 is the model of a manufacturing process with 2 machines $M_{1}$ and $M_{2}$ (corresponding to transitions $T_{1}$ and $T_{2}$ ) in a single production line. Machines are fed by buffers with limited capacities corresponding to the subsets of places $\left\{P_{1}, P_{1}^{\prime}\right\}$ and $\left\{P_{2}, P_{2}^{\prime}\right\}$. The maximal capacities $C_{1}$ and $C_{2}$ of the buffers correspond to the initial marking $m_{1}(0)+$ $m^{\prime}{ }_{1}(0)=C_{1}$ and $m_{2}(O)+m^{\prime}{ }_{2}(O)=C_{2}$. Pieces enter in the system by firing $T_{0}$. The number of pieces that are simultaneously processed by each machine is bounded by the marking of the places $P{ }_{0}, P{ }_{1}$, and $P{ }_{2}$. (i.e. an initial marking $m{ }_{i}(0)=1, i=0, \ldots, 2$ stands for single servers and $m{ }_{i}(0)>1$ stands for multi servers). 


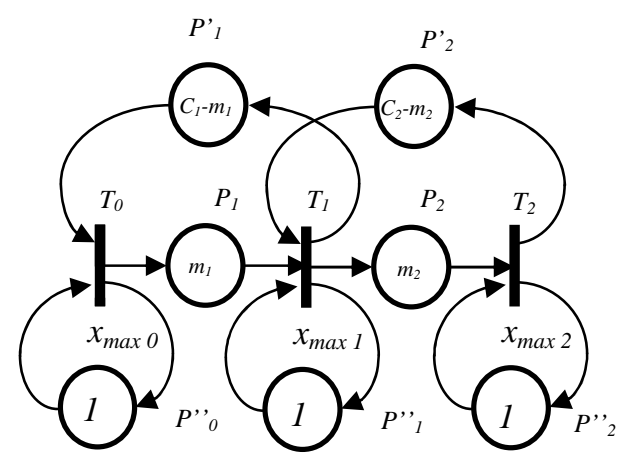

Figure 4: contPN model of a manufacturing process (system B)

[Insert figure 4 here]

The variations of controlled contPN (at this stage all transition are assumed to be potentially controllable) are written in scalar form (16) by using the functions $f_{j}$ defined as in (4):

$$
\begin{array}{lc}
\dot{m}_{1}(t)=x_{\max 0} \cdot u_{0}(t) \cdot m_{f 0}(t)-x_{\max 1} \cdot u_{1}(t) \cdot m_{f 1}(t) \\
\dot{m}_{2}(t)=x_{\max 1} \cdot u_{1}(t) \cdot m_{f 1}(t)-x_{\max 2} \cdot u_{2}(t) \cdot m_{f 2}(t) \\
m_{i}^{\prime}(t)=C_{i}-m_{i}(t) & i=1,2 \\
m^{\prime \prime}{ }_{h}(t)=m^{\prime \prime}{ }_{h}(0) & h=0,1,2
\end{array}
$$

Let us now introduce the outputs $y_{1}(t)=m_{1}(t)$, and $y_{2}(t)=m_{2}(t)$. The output $W$-sensitivity matrix is given by equation (17):

$$
\Sigma_{W}(B)=\left(\begin{array}{ccc}
u_{0} & u_{1} & u_{2} \\
0 & 0 & 1 \\
1 & 0 & 0
\end{array}\right) y_{1}
$$

The output $W$-sensitivity matrix $\Sigma_{W}(B)$ shows that the marking of each output depends on the firing of all transitions: the content of each intermediate buffer depends of the production rate of upstream but also downstream machines. But in order to drive the output $y_{1}$ it is more convenient to control transition $T_{0}$ or $T_{1}$ (rank - 0 inputs) than $T_{2}$ (rank -1 inputs). To drive the output $y_{2}$ it is more convenient to control transition $T_{1}$ or $T_{2}$ than $T_{0}$. Table 1 provides the output $W$-sensitivity matrices of several output configurations. 


\begin{tabular}{|c|c|c|c|c|}
\hline & $Y(t)=m_{l}(t)$ & $Y(t)=m_{2}(t)$ & $Y(t)=m_{l}(t)+m_{2}(t)$ & $Y(t)=\left(m_{1}(t), m_{2}(t)\right)^{T}$ \\
\hline$\Sigma_{W}$ & $Q_{l}=\left(\begin{array}{lllllll}0 & 0 & 0 & 1 & 0 & 0 & 0\end{array}\right)$ & $Q_{2}=\left(\begin{array}{lllllll}0 & 0 & 0 & 0 & 1 & 0 & 0\end{array}\right)$ & $Q_{3}=\left(\begin{array}{lllllll}0 & 0 & 0 & 1 & 1 & 0 & 0\end{array}\right)$ & $Q_{4}=\left(\begin{array}{lllllll}0 & 0 & 0 & 1 & 0 & 0 & 0 \\
0 & 0 & 0 & 0 & 1 & 0 & 0\end{array}\right)$ \\
\hline System B & $\left(\begin{array}{lll}0 & 0 & 1\end{array}\right)$ & $\left(\begin{array}{lll}1 & 0 & 0\end{array}\right)$ & $\left(\begin{array}{lll}0 & 0 & 0\end{array}\right)$ & $\left(\begin{array}{lll}0 & 0 & 1 \\
1 & 0 & 0\end{array}\right)$ \\
\hline System B' & $\left(\begin{array}{lll}0 & 0 & \infty\end{array}\right)$ & $\left(\begin{array}{lll}1 & 0 & 0\end{array}\right)$ & $\left(\begin{array}{lll}0 & 0 & 0\end{array}\right)$ & $\left.\begin{array}{ccc}0 & 0 & \infty \\
1 & 0 & 0\end{array}\right)$ \\
\hline
\end{tabular}

Table 1: Output $W$ - sensitivity matrices for systems B and B'

[Insert table 1 here]

The investigation of the causality relationships is useful in order to design efficient control. For instance, if the controller goal is to reach a desired level in first intermediate buffer or to track a desired trajectory, it is more convenient to control the input transition $T_{0}\left(\sigma_{W 10}=0\right)$, than the transition $T_{2}\left(\sigma_{W 12}=1\right)$. Such a conclusion will be confirmed in section 4 .

The results obtained with the structural analysis are more explicit with a modification of the previous example. The system B is changed in B' such that the intermediate buffers, used to store products after each operation, have an infinite capacity according to figure 5 and equation (18):

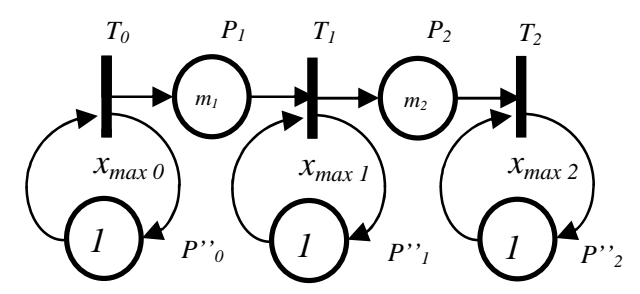

Figure 5: contPN model of system B'

[Insert figure 5 about here]

$$
\begin{array}{lr}
\dot{m}_{1}(t)=x_{\max 0} \cdot u_{0}(t) \cdot m^{\prime \prime}(t)-x_{\max 1} \cdot u_{1}(t) \cdot m_{f 1}(t) \\
\dot{m}_{2}(t)=x_{\max 1} \cdot u_{1}(t) \cdot m_{f 1}(t)-x_{\max 2} \cdot u_{2}(t) \cdot m_{f 2}(t) \\
m{ }^{\prime \prime}{ }_{h}(t)=m{ }^{\prime \prime}{ }_{h}(0) & h=0,1,2
\end{array}
$$


The output $W$-sensitivity matrix $\Sigma_{W}\left(B^{\prime}\right)$ is given by (19):

$$
\Sigma_{W}\left(B^{\prime}\right)=\left(\begin{array}{ccc}
u_{0} & u_{1} & u_{2} \\
0 & 0 & \infty \\
1 & 0 & 0
\end{array}\right) y_{1}
$$

From this matrix, it is obvious that transition $T_{2}$ can no longer be used to control the output $y_{1}(t)=m_{1}(t)$ : there exists no causality relationship from $T_{2}$ to $P_{1}$ because of the infinite capacity buffer represented by $P_{2}$. The $W$-sensitivity matrices obtained for several output configurations must be compared with system B.

Another example of contPN is given by system $\mathrm{C}$ in figure 6 with the marking vector $M(t)=$ $\left(m_{1}{ }_{1}(t), m_{2}{ }_{2}(t), m^{\prime}{ }_{3}(t), m^{\prime}{ }_{4}(t), m^{\prime}{ }_{5}(t), m_{1}(t), m_{2}(t), m_{3}(t), m_{4}(t)\right)^{\mathrm{T}}$. Weighted $\operatorname{arcs} T_{2} \rightarrow P_{1}$ and $P_{1}$ $\rightarrow T_{1}$ means that the flow of tokens that fire $T_{2}$ to $P_{1}$ is multiplied by 2 and the flow of tokens that fire $T_{1}$ from $P_{1}$ is divided by 3 . As previously, places $P^{\prime}{ }_{1}$ to $P^{\prime}{ }_{5}$ limit the number of simultaneous firings of the transitions $T_{1}$ to $T_{5}$. The outputs are defined according to $y_{1}(t)=$ $m_{1}(t)+m_{3}(t)$ and $y_{2}(t)=m_{2}(t)+m_{4}(t)$.

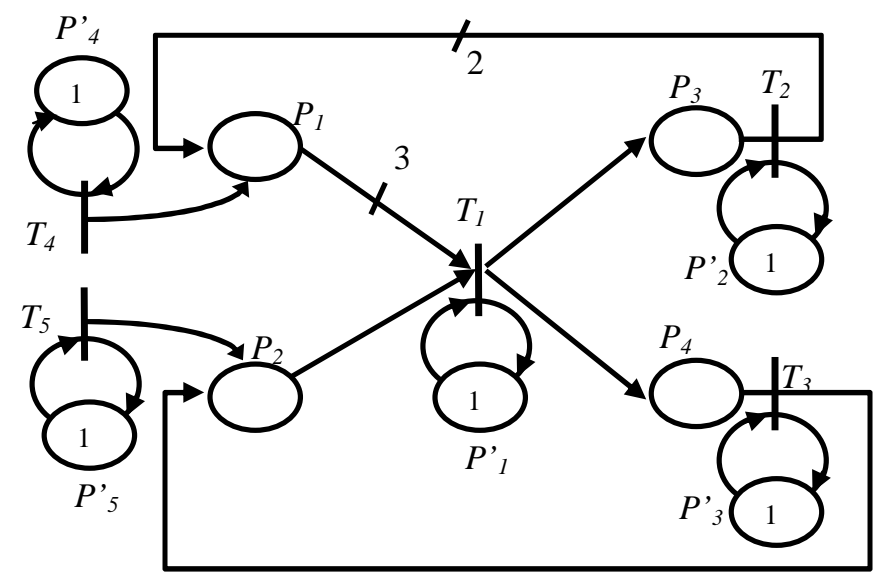

Figure 6: Closed loop process (system C)

[Insert figure 6 here]

The controlled contPN is written in scalar form (20): 


$$
\begin{aligned}
& \dot{m}_{1}(t)=x_{\max 4} \cdot u_{4}(t)+2 \cdot x_{\max 2} \cdot u_{2}(t) \cdot m_{f 2}(t)-3 \cdot\left(\frac{x_{\max 1}}{w_{f 11}^{P R}}\right) \cdot u_{1}(t) \cdot m_{f 1}(t) \\
& \dot{m}_{2}(t)=x_{\max 5} \cdot u_{5}(t)+x_{\max 3} \cdot u_{3}(t) \cdot m_{f 3}(t)-\left(\frac{x_{\max 1}}{w_{f 11}^{P R}}\right) \cdot u_{1}(t) \cdot m_{f 1}(t) \\
& \dot{m}_{3}(t)=\left(\frac{x_{\max 1}}{w_{f 11}^{P R}}\right) \cdot u_{1}(t) \cdot m_{f 1}(t)-x_{\max 2} \cdot u_{2}(t) \cdot m_{f 2}(t) \\
& \dot{m}_{4}(t)=\left(\frac{x_{\max 1}}{w_{f 11}^{P R}}\right) \cdot u_{1}(t) \cdot m_{f 1}(t)-x_{\max 3} \cdot u_{3}(t) \cdot m_{f 3}(t)
\end{aligned}
$$

Let us mention that the functions $f_{4}$ and $f_{5}$ are constant and $m_{f 4}(t)=m_{f 5}(t)=1$. The inputoutput $W$-sensitivity matrix (21) shows that both outputs are correlated according to the transition $T_{1}$. Another conclusion is that the set of transitions $\boldsymbol{T}_{\boldsymbol{C}}=\left\{T_{4}, T_{5}\right\}$ or $\boldsymbol{T}_{\boldsymbol{C}}=\left\{T_{2}, T_{3}\right\}$ are reasonable choice to drive the outputs $y_{1}$ and $y_{2}$ due to the difference in the sensitivity ranks.

$$
\begin{aligned}
& \begin{array}{llllll}
u_{1} & u_{2} & u_{3} & u_{4} & u_{5}
\end{array} \\
& \Sigma_{W}(C)=\left(\begin{array}{lllll}
0 & 0 & 1 & 0 & 1 \\
0 & 1 & 0 & 1 & 0
\end{array}\right) \begin{array}{l}
y_{1} \\
y_{2}
\end{array}
\end{aligned}
$$

To conclude output $W$-sensitivity is helpful to select the transitions to be controlled wrt a set of output configurations. This structural analysis will be used in the next section to design the sets $\boldsymbol{T}_{\boldsymbol{C}}$ used with GBC.

\section{Control design for contPN}

Flow control for contPN was investigated by several authors (Giua et al. 2006, Mahulea et al. 2008b, Julvez and Boel 2010, Kara et al. 2009, Vazquez and Silva 2009, Apaydin-Ozkan et al. 2011, Wang et al. 2013). Such methods have provided interesting results but require strong conditions concerning the transitions to control and the places to observe. In particular, in many existing works all transitions are usually assumed to be controlled. This paper focuses on another approach based on gradient method suitable for contPN where all transitions are not controllable. Gradient-based methods have been intensively investigated for the learning of neural networks (Widrow and Lehr 1990) and the identification of continuous systems (Thomas 1997) but only a few studies have concerned the hybrid and discrete event systems (Balduzzi et al. 2000). This approach takes advantages on the propagation of the gradient 
through the contPN nodes in order to minimise the quadratic instantaneous error between desired and measured outputs by slowing down the activity of controllable transitions. Gradient algorithms perform the minimisation of a scalar cost function that evaluates the distance between the desired output $Y^{d e s}(t)$ and the system output $Y(t)$.

\subsection{Sensitivity functions}

Gradient algorithms are based on the evaluation of sensitivity functions. Such functions are defined for contPN (definition 4.1) and their variation is expressed with differential equations (theorem 4.1).

Definition 4.1: The marking sensitivity function $s_{i \gamma}(t)$ of the marking $m_{i}$ wrt the input $u_{\gamma}$ of any transition $T_{\gamma} \in \boldsymbol{T}$ and the output sensitivity function $\sigma_{\alpha \gamma}(t)$ of the output $y_{\alpha}$ wrt the input $u_{\gamma}$ are defined as (22):

$$
s_{i \gamma}(t)=\frac{\partial m_{i}(t)}{\partial u_{\gamma}}, \quad \sigma_{\alpha \gamma}(t)=\frac{\partial y_{\alpha}(t)}{\partial u_{\gamma}}=\sum_{i=1}^{n}\left(q_{\alpha i} s_{i \gamma}(t)\right)
$$

As explained in part 2.3, $u_{\gamma}(t)$ is constant and equal to 1 for non controllable transitions $(T \in$ $\left.\boldsymbol{T}_{N C}\right)$. The variation of any scalar sensitivity function $s_{i \gamma}(t)$ for $i=1, \ldots, n$ and $\gamma=1, \ldots, q$ is given by (23):

$$
\begin{aligned}
& \frac{d s_{i \gamma}(t)}{d t}=\frac{d}{d t}\left(\frac{\partial m_{i}(t)}{\partial u_{\gamma}}\right)=\frac{\partial}{\partial u_{\gamma}}\left(\frac{d m_{i}(t)}{d t}\right)=\sum_{j=1}^{q} w_{i j} \cdot \frac{\partial x_{C j}(t)}{\partial u_{\gamma}} \\
& \frac{d s_{i \gamma}(t)}{d t}=\left(\frac{w_{i \gamma} \cdot x_{\max \gamma}}{w^{P R}{ }_{f \gamma \gamma}}\right) \cdot m_{f \gamma}(t)+\sum_{j=1}^{q}\left(\frac{w_{i j} \cdot x_{\max j}}{w^{P R}{ }_{f j}}\right) \cdot u_{j}(t) \cdot \frac{\partial m_{f j}(t)}{\partial u_{\gamma}} \\
& \frac{d s_{i \gamma}(t)}{d t}=\left(\frac{w_{i \gamma} \cdot x_{\max \gamma}}{w^{P R}{ }_{f \gamma \gamma}}\right) \cdot m_{f \gamma}(t)+\sum_{j=1}^{q}\left(\frac{w_{i j} \cdot x_{\max j}}{w^{P R}{ }_{f j}}\right) \cdot u_{j}(t) \cdot s_{f j \gamma}(t)
\end{aligned}
$$

The marking and output sensitivity vectors $S_{\gamma}(t)=\left(s_{i \gamma}\right) \in \mathbf{R}^{n}$ and $\Sigma_{\gamma}(t)=\left(\sigma_{\alpha \gamma}\right) \in \mathbf{R}^{e}$ of marking $M(t)$ and output $Y(t)$ wrt input $u_{\gamma}$ are defined from definition 4.1. Let us denote $W(\therefore, \gamma)$ as the column $\gamma$ in matrix $W$ and $A_{\varphi}(\gamma,:)$ as the row $\gamma$ in matrix $A_{\varphi}$ One can remark that $m_{f \gamma}(t) / w^{P R}{ }_{f \gamma \gamma}$ corresponds to $A_{\varphi}(\gamma,:) . M(t)$ when $M(t)$ is in region $\mathbf{A}_{\varphi}$. Equation (23) is rewritten in vectorial form and the variations of sensitivity vectors $S_{\gamma}(t)$ and $\Sigma_{\gamma}(t)$, wrt input $u_{\gamma}$ are given for all $M(t)$ $\in \mathbf{A}_{\varphi}$ by equation (24): 


$$
\begin{aligned}
& \frac{d S_{\gamma}(t)}{d t}=x_{\max \gamma} \cdot W(:, \gamma) \cdot A_{\varphi}(\gamma,:) \cdot M(t)+W \cdot X_{\max } \cdot \operatorname{diag}(U(t)) \cdot A_{\varphi} \cdot S_{\gamma}(t), \quad \gamma=1, \ldots, q \\
& S_{\gamma}(0)=0 \\
& \Sigma_{\gamma}(t)=Q \cdot S_{\gamma}(t)
\end{aligned}
$$

All sensitivity functions and vectors are summed up in sensitivity matrices:

$$
S(t)=\left(S_{1}(t)|\ldots| S_{q}(t)\right)=\left(s_{i \gamma}(t)\right) \in \mathbf{R}^{n \times q}
$$

$\Sigma(t)=Q . S(t)=\left(\Sigma_{1}(t)|\ldots| \Sigma_{q}(t)\right)=\left(\sigma_{\alpha \gamma}(t)\right) \in \mathbf{R}^{e \times q}$

and $\left(S_{i *}(t)\right)^{T} \in \mathbf{R}^{1 \times q}\left(\operatorname{resp} .\left(\Sigma_{\alpha^{*}}(t)\right)^{T} \in \mathbf{R}^{1 \times q}\right)$ stands for the row $i$ of matrix $S(t)$ (resp. row $\alpha$ of matrix $\Sigma(t))$. Each column of the sensitivity matrices corresponds to the sensitivity of a given variable wrt the control action for all transitions and each row of the sensitivity matrices corresponds to the sensitivity of all markings and outputs wrt to a given input.

\subsection{Discrete time approximation for numerical issues}

For numerical issues, let us introduce the sampling period $\Delta t$ and a first order approximation of the variation equations. The sampling period $\Delta t$ is selected to be small enough in comparison with the magnitude of eigenvalues of the matrices $W . X_{\max } \cdot A_{\varphi}, \varphi=1, \ldots, K$. In addition, the sampling period $\Delta t$ satisfies (27) for all places $P_{i} \in \mathbf{P}$ :

$$
\left(\sum_{T_{j} \in P_{i}^{\circ}} x_{\max j}\right) \cdot \Delta t<1,
$$

such that any reachable marking with discrete time trajectory is non-negative (Mahuela et al., 2008b). In the following, $k>0$ refers to the discrete time $t=k . \Delta t$. Equations (12) and (13) lead to:

$$
\begin{aligned}
& m_{i}(k+1)=m_{i}(k)+\Delta t \cdot\left(\sum_{j=1}^{q}\left(\frac{w_{i j} \cdot x_{\max j}}{w_{f j j}^{P R}}\right) \cdot u_{j}(k) \cdot m_{f_{j}}(k)\right), \quad i=1, \ldots, n \\
& y_{\alpha}(k)=\sum_{i=1}^{n} q_{\alpha i} \cdot m_{i}(k), \quad \alpha=1, \ldots, e
\end{aligned}
$$

For all $M(k) \in \mathbf{A}_{\varphi}$ : 


$$
\begin{aligned}
& M(k+1)=\left(I_{n}+\Delta t \cdot W_{C} \cdot X_{\text {max }} \cdot \operatorname{diag}(U(k)) \cdot A_{\varphi}\right) \cdot M(k) \\
& Y(k)=Q \cdot M(k)
\end{aligned}
$$

where $I_{n}$ stands for the identity matrix of dimension $n \times n$. Similarly equations (24) and (23) lead to:

$$
s_{i \gamma}(k+1)=s_{i \gamma}(k)+\Delta t .\left(\frac{w_{i \gamma} \cdot x_{\max \gamma}}{w_{f \gamma \gamma}^{P R}}\right) \cdot m_{f \gamma}(k)+\Delta t \cdot\left(\sum_{j=1}^{q}\left(\frac{w_{i j} \cdot x_{\max j}}{w_{f j j}^{P R}}\right) \cdot u_{j}(k) \cdot s_{f j \gamma}(k)\right), \quad i=1, . ., n, \quad \gamma=1, \ldots, q
$$

$$
S_{\gamma}(k+1)=\left(I_{n}+\Delta t \cdot W \cdot X_{\max } \cdot \operatorname{diag}(U(k)) \cdot A_{\varphi}\right) \cdot S_{\gamma}(k)+\Delta t \cdot x_{\max \gamma} \cdot W(:, \gamma) \cdot A_{\varphi}(\gamma,:) \cdot M(k), \quad \gamma=1, \ldots, q
$$

\subsection{Gradient-based controllers}

For the seek of simplicity, let us first consider the case of the single output $y_{d}(k)$. The instantaneous error at instant $k$ and at step $i$ is defined as $\varepsilon_{d}(k, i)=y_{d e s}^{d}(k)-y_{d}(k, i)$, where $y^{d e s}(k)$ stands for the $\alpha^{\text {th }}$ desired output and $y_{d}(k, i)$ stands for the $\alpha^{\text {th }}$ actual output obtained from the marking $M(k)$ and from the input vector $U(k, i)$ :

$$
y_{\alpha}(k, i)=\sum_{i=1}^{n} q_{\alpha i} \cdot\left(m_{i}(k)+\Delta t \cdot\left(\sum_{j=1}^{q}\left(\frac{w_{i j} \cdot x_{\max j}}{w_{f j j}^{P R}}\right) \cdot u_{j}(k, i) \cdot m_{f_{j}}(k)\right)\right), \quad \alpha=1, \ldots, e
$$

$U(k, i)$ is the updating of the input vector, at time $k$, obtained after the $i^{\text {th }}$ iteration of the algorithm described below. Let us consider the scalar cost function $v_{\alpha}(k, i)$ to be minimized:

$$
v_{\alpha}(k, i)=\frac{1}{2}\left(\varepsilon_{\alpha}(k, i)\right)^{2} \in \mathbf{R}^{+}
$$

The proposed controller results from the Taylor series expansion of the cost function $v_{d}(k, i)$ in the neighbourhood of $U(k, i)$ :

$$
\begin{aligned}
& v_{\alpha}(k, i+1)=v_{\alpha}(k, i)+\left(\frac{\partial v_{\alpha}}{\partial U}\right)_{U=U(k, i)}^{T} \delta U(k, i) \\
& +\frac{1}{2} .(\delta U(k, i))^{T}\left(\frac{\partial^{2} v_{\alpha}}{\partial U . \partial U^{T}}\right)_{U=U(k, i)} \delta U(k, i)+o\left(\left|\delta U(k, i)^{T} . \delta U(k, i)\right|\right)
\end{aligned}
$$


with $\delta U(k, i)=U(k, i+1)-U(k, i)$. The optimal value of the control actions are worked out according to the stationary condition:

$$
\delta U(k, i)=-2 \cdot\left(\frac{\partial^{2} v_{\alpha}}{\partial U . \partial U^{T}}\right)_{U=U(k, i)}^{-1} \cdot\left(\frac{\partial v_{\alpha}}{\partial U}\right)_{U=U(k, i)}
$$

under the constraints $0 \leq u_{j}(t) \leq 1$ for $T \in \boldsymbol{T}_{\boldsymbol{C}}$ and $u_{j}(t)=1$ for $T \in \boldsymbol{T}_{N C}$ and the sets $\boldsymbol{T}_{\boldsymbol{C}}$ is determined according to the $W$-sensitivity. Using the sensitivity vector $\Sigma_{\alpha^{*}}(t)$ introduced in section 4.1 and worked out at time $k$, one can write:

$$
\begin{aligned}
& \left(\frac{\partial v_{\alpha}}{\partial U}\right)_{U=U(k, i)}=-\Sigma_{\alpha^{*}}(k) \cdot \varepsilon_{\alpha}(k, i) \\
& \left(\frac{\partial^{2} v_{\alpha}}{\partial U \cdot \partial U^{T}}\right)_{U=U(k, i)} \approx\left(\frac{\partial^{2} v_{\alpha}}{\partial U \cdot \partial U^{T}}\right)_{U=U(k-1)} \approx \Sigma_{\alpha^{*}}(k) \cdot \Sigma_{\alpha^{*}}(k)^{T}+\theta \cdot I_{q}
\end{aligned}
$$

where the term $\theta \cdot I_{q}$ is added in order to approximate the inverse of the Hessien matrix when it is not regular or badly conditioned (Hagan et al. 1995). Let us notice that second order terms are neglected in the computation of (36) and the sensitivity functions do not depend on the iteration $i: \Sigma_{\alpha^{*}}(k)$ is computed a single time for each new measurement. Thus, equation (34) results in the updating rule of the controller (37):

$$
\begin{aligned}
& U(k, i+1)=U(k, i)+2 \cdot\left(\Sigma_{\alpha^{*}}(k) \cdot \Sigma_{\alpha^{*}}(k)^{T}+\theta \cdot I_{q}\right)^{-1} \cdot \Sigma_{\alpha^{*}}(k) \cdot \varepsilon_{\alpha}(k, i), i=0, \ldots, N-1 \\
& U(k, 0)=U(k-1)
\end{aligned}
$$

under the constraints $0 \leq u_{j}(t) \leq 1$ for $T \in \boldsymbol{T}_{\boldsymbol{C}}$ and $u_{j}(t)=1$ for $T \in \boldsymbol{T}_{\boldsymbol{N C}}$. A maximal number of $N$ iterations is considered, for each instant $k$ in order to work out the control actions in a finite number of steps consistent with real time constraints. According to this truncation, we have $U(k)=U(k, N)$.

Let us point out two limit cases. When $\theta>>1$, equation (37) corresponds to the gradient method (Van der Smagt et al. 1994):

$U(k, i+1)=U(k, i)+\frac{2}{\theta} \cdot \Sigma_{\alpha^{*}}(k) \cdot \varepsilon_{\alpha}(k, i), i=0, \ldots, N-1$. 
When $\theta<<1$, equation (37) corresponds to the Gauss-Newton method (Thomas 1997):

$$
U(k, i+1)=U(k, i)+2 \cdot\left(\Sigma_{\alpha^{*}}(k) \cdot \Sigma_{\alpha^{*}}(k)^{T}\right)^{-1} \cdot \Sigma_{\alpha^{*}}(k) \cdot \varepsilon_{\alpha}(k, i), i=0, \ldots, N-1
$$

The previous controller can be generalised in the multi-outputs case, by considering the error vector $E(k, i)=Y^{d e s}(k)-Y(k, i)$, and the scalar cost function (40):

$$
v(k, i)=\frac{1}{2} \cdot E^{T}(k, i) \cdot E(k, i) \in \mathbf{R}
$$

that results in the following updating rule for the controller:

$$
\begin{aligned}
& U(k, i+1)=U(k, i)+2 \cdot\left(\Sigma^{T}(k) \cdot \Sigma(k)+\theta \cdot I_{q}\right)^{-1} \cdot \Sigma^{T}(k) \cdot E(k, i), i=0, \ldots, N-1 \\
& U(k, 0)=U(k-1)
\end{aligned}
$$

under the constraints $0 \leq u_{j}(t) \leq 1$ for $T \in \boldsymbol{T}_{C}$ and $u_{j}(t)=1$ for $T \in \boldsymbol{T}_{N C}$.

\section{Examples}

In all simulations, the sampling period is $\Delta t=0.1$ and the parameter is $\theta=0.1$ in order to avoid the singularities in the Hessien approximation (34).

\subsection{ContPN with a single controllable transition}

Let us first consider contPN B (figure 4) with incidence matrices and parameters defined as in section 3, initial marking vector $M_{I}=\left(\begin{array}{lllllll}1 & 1 & 1 & 0 & 0 & 3 & 3\end{array}\right)^{\mathrm{T}}$, maximal firing rates matrix $X_{\max }=$ $\operatorname{diag}(5,4,3)$ and $\boldsymbol{T}_{\boldsymbol{C}}=\left\{T_{0}\right\}$. Figure 7 points out the influence of the output matrix on the controller response: three scalar outputs are investigated $y_{1}(t)=m_{1}(t), y_{2}(t)=m_{2}(t)$ and $y_{3}(t)=$ $m_{1}(t)+m_{2}(t)$ that correspond respectively to $Q_{1}=\left(\begin{array}{lllllll}0 & 0 & 0 & 1 & 0 & 0 & 0\end{array}\right), Q_{2}=\left(\begin{array}{lllllll}0 & 0 & 0 & 0 & 1 & 0 & 0\end{array}\right)$ and $Q_{3}=$

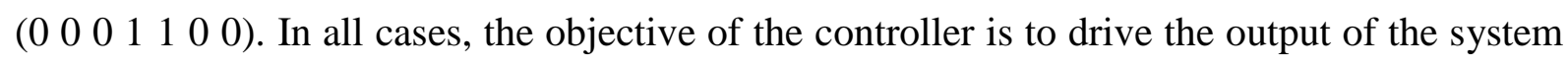
to the desired value $y^{\text {des }}=2$ tokens. The maximal number of iterations is $N=100$. For each case, figure 7 presents the control actions for transition $T_{0}$, the transition flows for $T_{0}$, the output trajectories and the output errors. Concerning the output matrices $Q_{1}$ and $Q_{3}$ the desired value is rapidly reached with a good accuracy, but in case of output matrix $Q_{2}$ oscillations are observed that result from the controller that tries to compensate the oscillation around the desired value. Such an input - output specification is not suitable with our approach because the marking of the unobservable place $P_{1}$ is not considered in the calculation of the input firing frequency. As a consequence, the desired level is exceeded and 
oscillations arise due to the delay between the firing of $T_{0}$ and the observation of $P_{2}$ marking. In order to avoid the undesirable cumulative effects of the marking, the inputs and outputs of the systems must be preferred such that the sensitivity rank equals 0 (immediate neighbourhood) as shown in table 1.
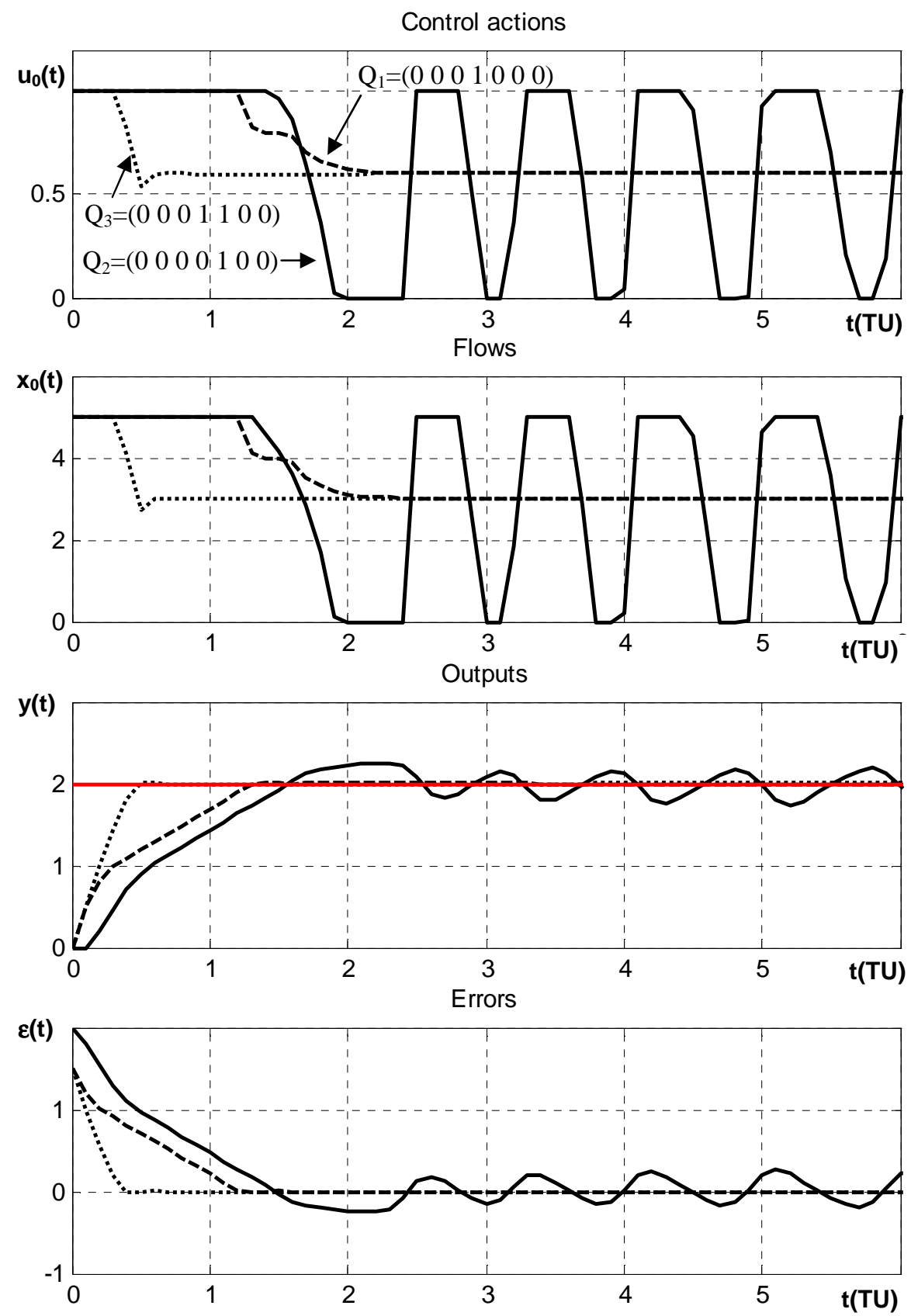

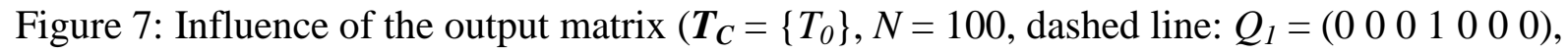

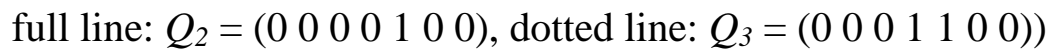

[Insert figure 7 here] 
The speed of the algorithm increases as the maximal number of iterations in the gradient based algorithm. Figure 8 illustrates the influence of the number of iterations $N$ when the output matrix is given by $Q_{1}=\left(\begin{array}{lllllll}0 & 0 & 0 & 1 & 0 & 0 & 0\end{array}\right)$. For a small number of iterations $(N=2)$, the controller is not quick enough to correct the output error. In comparison, a large number $(N=$ 100) compensates the slowness of the gradient algorithm.
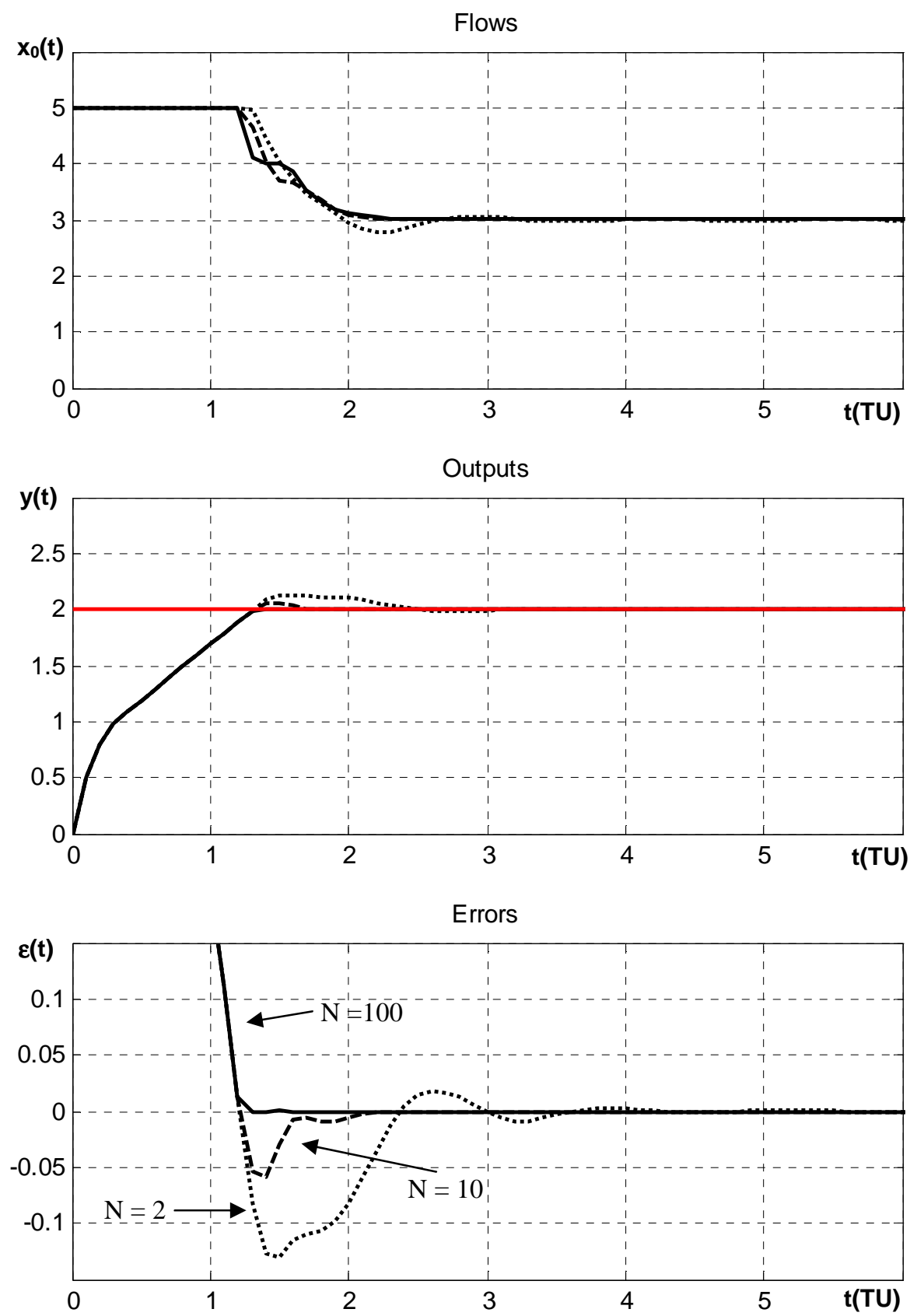

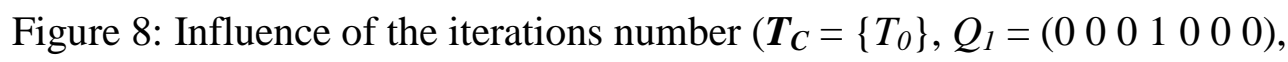
full line: $N=100$, dashed line: $N=10$, dotted line: $N=2$ ) 


\subsection{Comparison with other control methods}

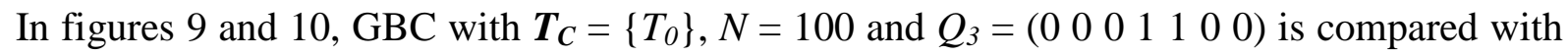
parallel Proportional Integral Controllers (PIC) with $K_{p}=10, K_{I}=0.2$, on/off and Model Predictive Controller (MPC) (Giua et al. 2006, Mahulea et al. 2008b) when the desired output is the piecewise linear function given by equation (42):

$$
\left\{\begin{array}{l}
y^{d e s}(t)=1,0 \leq t<4 \\
y^{d e s}(t)=|\sin (5 . t-6)|+1+\frac{t-6}{4}, \quad t \geq 4
\end{array}\right.
$$

This desired input is composed of two parts: the first one corresponds to a regulation problem during $4 \mathrm{TU}$ and the second one to a tracking one during the last $8 \mathrm{TU}$.
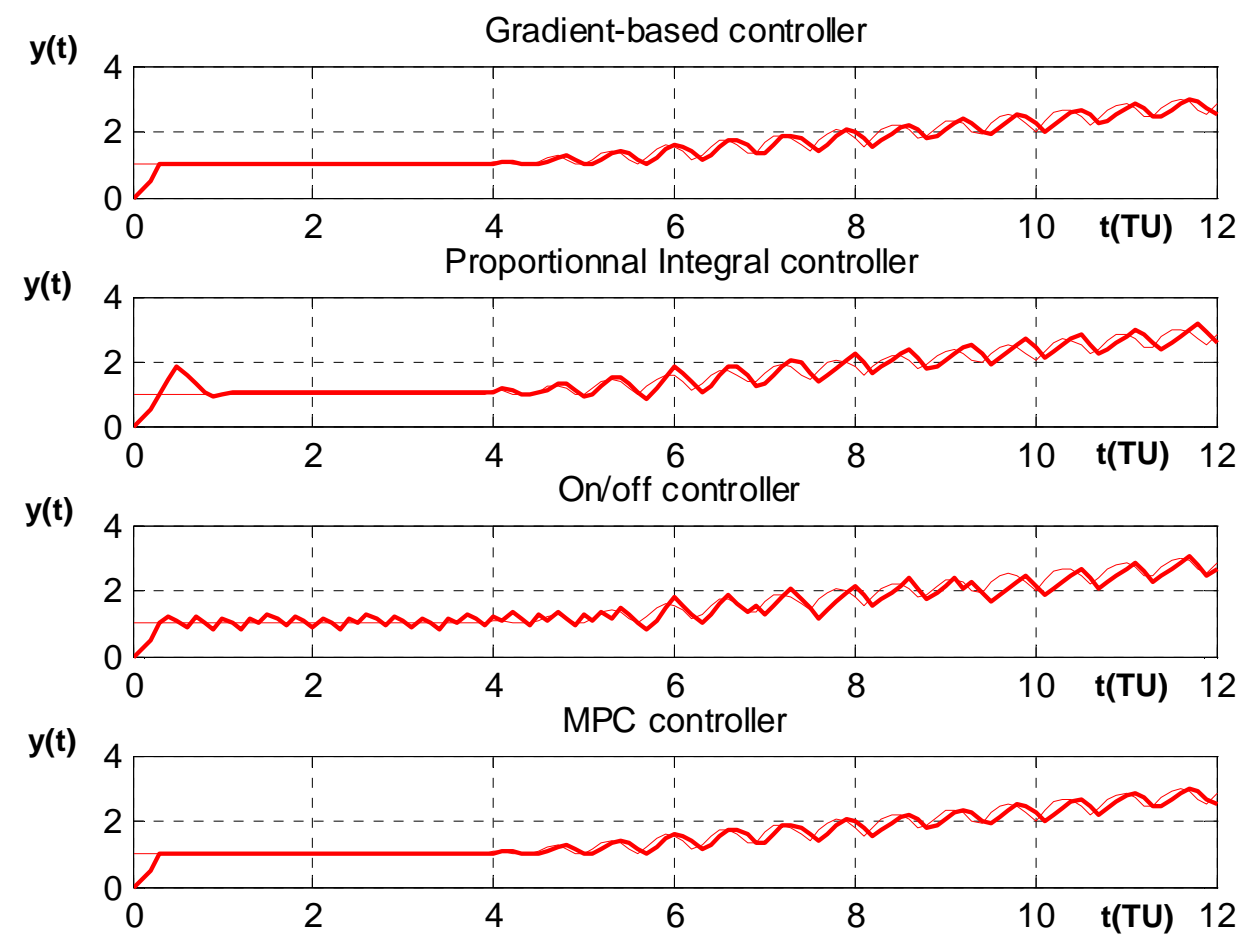

Figure 9: Control performances comparison:

Outputs and desired output (in bold) for GBC, PIC, on/off controller and MPC.

[Insert figure 9 here]

One can observed that the desired output is globally correctly tracked (regulation and tracking parts). The output signal is very different in function of the controller choice. The PIC uses the error signal as the input. As a consequence, this controller is not suitable when the error signal presents a lot of variations. The on/off controller is defined as a series of 
commutations. Generally speaking, the variations of the flows resulting from the GBC and MPC are smooth comparing to those given by the other controllers, in particular on/off controller. Both controllers, GBC and MPC are similar in this example (different performances occur according to the controlled transitions set (figure 10) both trajectories $\left(y(t)\right.$ and $\left.y^{\text {des }}(t)\right)$ are superimposed for these two controllers.

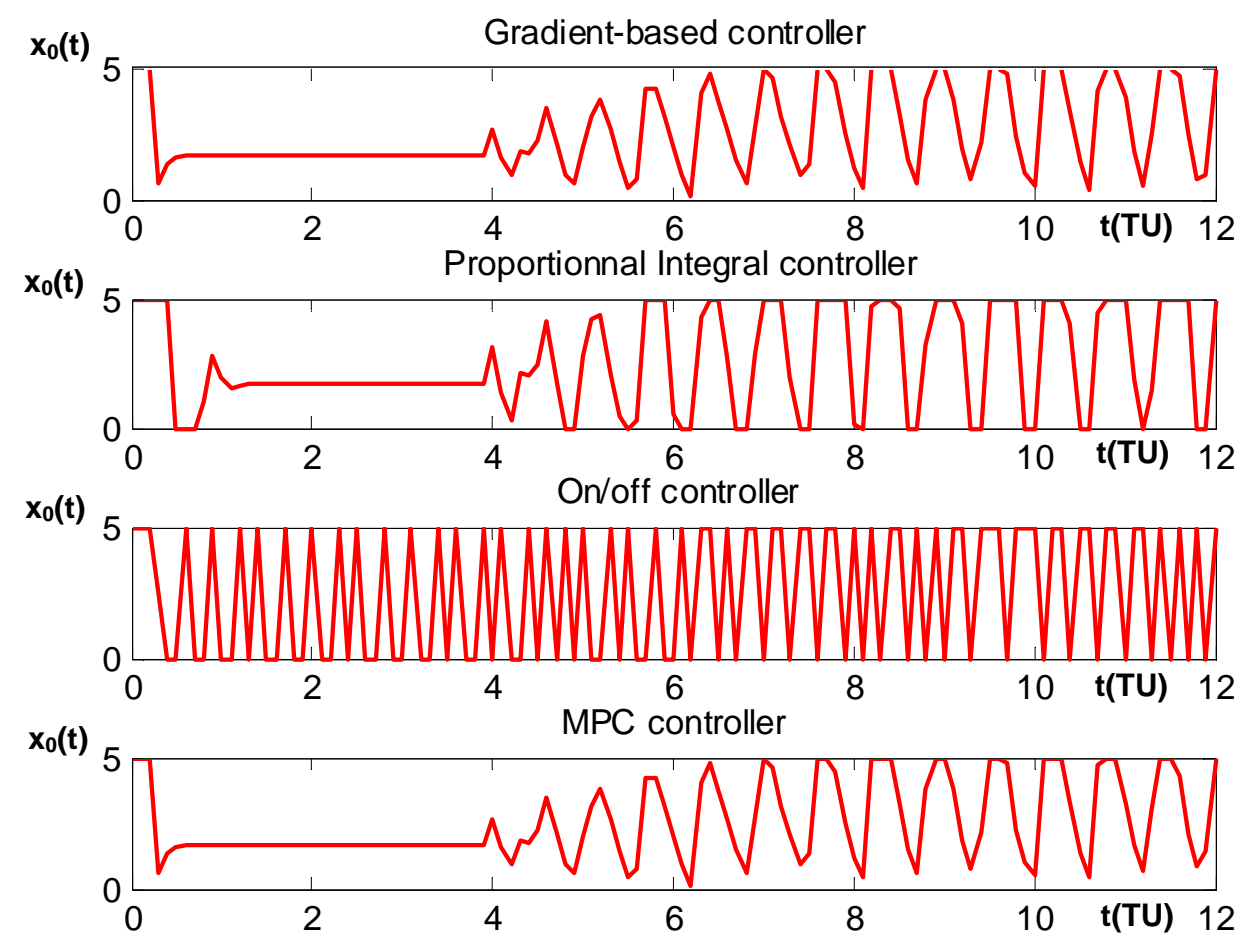

Figure 10: Control performance comparison:

Flows for GBC, PIC, on/off controller and MPC.

[Insert figure 10 here]

To compare these controllers, MSE are computed for the regulation problem and for the tracking one. The MSE is defined by (43):

$$
M S E=\frac{1}{t_{2}-t_{1}} \sum_{t_{1}}^{t_{2}}\left(Y^{d e s}(k)-Y(k)\right)^{T} \cdot\left(Y^{d e s}(k)-Y(k)\right),
$$

where $t_{1}$ and $t_{2}$ define either the regulation time interval $\left(t_{1}=0, t_{2}=3.9 \mathrm{UT}\right)$ or the tracking time interval $\left(t_{1}=4, t_{2}=12 \mathrm{UT}\right)$. 


\begin{tabular}{|l|c|c|}
\hline & Regulation: $t \in[0: 4[$ UT & Tracking: $t \in[4: 12]$ UT \\
\hline GBC & 0.032 & 0.038 \\
\hline PIC & 0.065 & 0.064 \\
\hline On/off & 0.054 & 0.08 \\
\hline MPC & 0.032 & 0.036 \\
\hline
\end{tabular}

Table 2: Comparison of controller performance

[Insert table 2 here]

All proposed controllers track the desired trajectories with a mean square error that does not exceed 0.08 token. Let us first notice the GBC and the MPC give very similar results, the MSE of the GBC is smaller than the one of the PIC or the on/off controller.

To refine the comparison between GBC and MPC, not only $Q_{3}$ but also $Q_{1}$ and $Q_{2}$ are used with the same desired output (figure 11). Only $T_{0}$ is controllable.
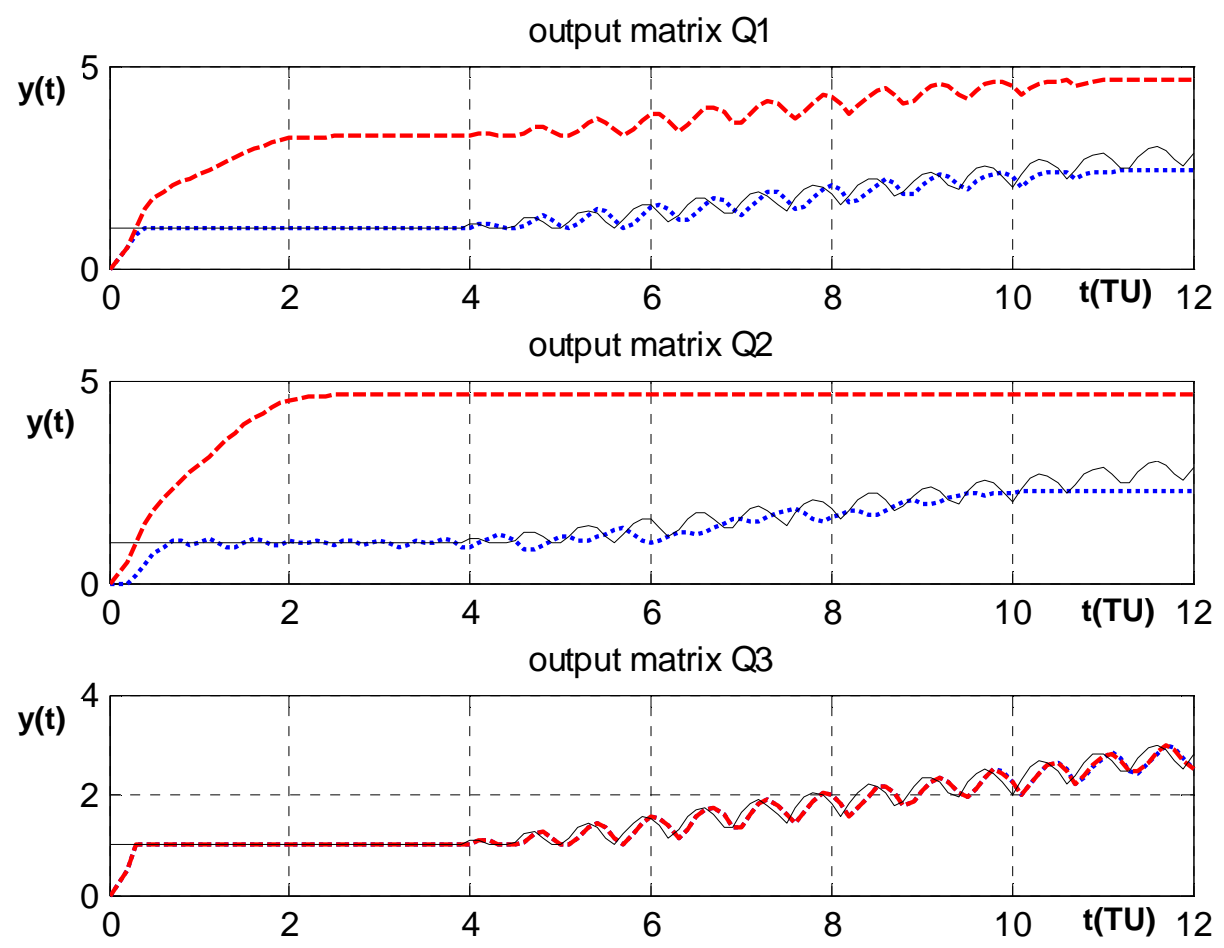

Figure 11: Comparison between GBC (dotted line) and MPC (dashed line) for $Q_{1}, Q_{2}$ and $Q_{3}$. [Insert figure 11 here] 
For the three cases, during the regulation part, GBC reaches the desired output while the MPC fails with output matrices $Q_{1}$ and $Q_{2}$. Although GBC presents some oscillations with the output matrix $Q_{2}$, it provides a better tracking than MPC with uncontrollable transitions. Concerning the tracking period, GBC follows the mean value of the desired output in spite of uncontrollable transitions. The same result is obtained between GBC and MPC with output matrices $Q_{13}$.

\subsection{ContPN with several controllable transitions}

Let us now consider the contPN C (figure 6) with $\boldsymbol{T}_{\boldsymbol{C}}=\left\{T_{4}, T_{5}\right\}$ and two outputs that correspond to the marking of the subsets of places $\left\{P_{1}, P_{3}\right\}$ and $\left\{P_{2}, P_{4}\right\}$ (i.e. $Q=\left(\begin{array}{llllll}0 & 0 & 0 & 0 & 0 & 1\end{array}\right.$

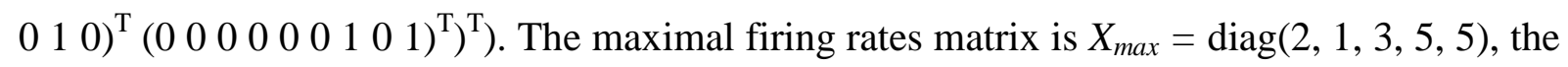

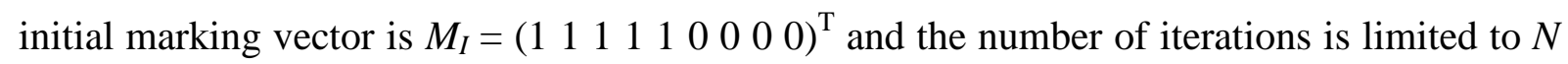
$=100$. The desired trajectories correspond to two piecewise linear trajectories given by equation (44):

$$
\left\{\begin{array} { l } 
{ y ^ { d e s } ( t ) = \frac { 5 } { 3 } \cdot t , \quad 0 \leq t < \frac { 1 2 } { 5 } } \\
{ y ^ { d e s } ( t ) = \frac { 5 } { 2 4 } \cdot t + \frac { 7 } { 2 } , \quad t \geq \frac { 1 2 } { 5 } }
\end{array} \quad \left\{\begin{array}{l}
y^{\text {des }}{ }_{2}(t)=t, \quad 0 \leq t<6 \\
y^{\text {des }}{ }_{2}(t)=\frac{1}{3} \cdot t+4, \quad t \geq 6
\end{array}\right.\right.
$$

The outputs of the GBC are presented in figure 12. In comparison, PIC and on/off controllers provide only poor results because the inputs and outputs of the system $\mathrm{C}$ are coupled thanks to the transition $T_{1}$. These controllers focus on one desired trajectory but cannot track simultaneously both ones. On the contrary, the GBC tracks simultaneously both trajectories with an instantaneous error that does not exceed 0.005 tokens. The input-output decomposition is obtained thanks to the sensitivity functions that evaluate for each output the relative influence of both inputs. 

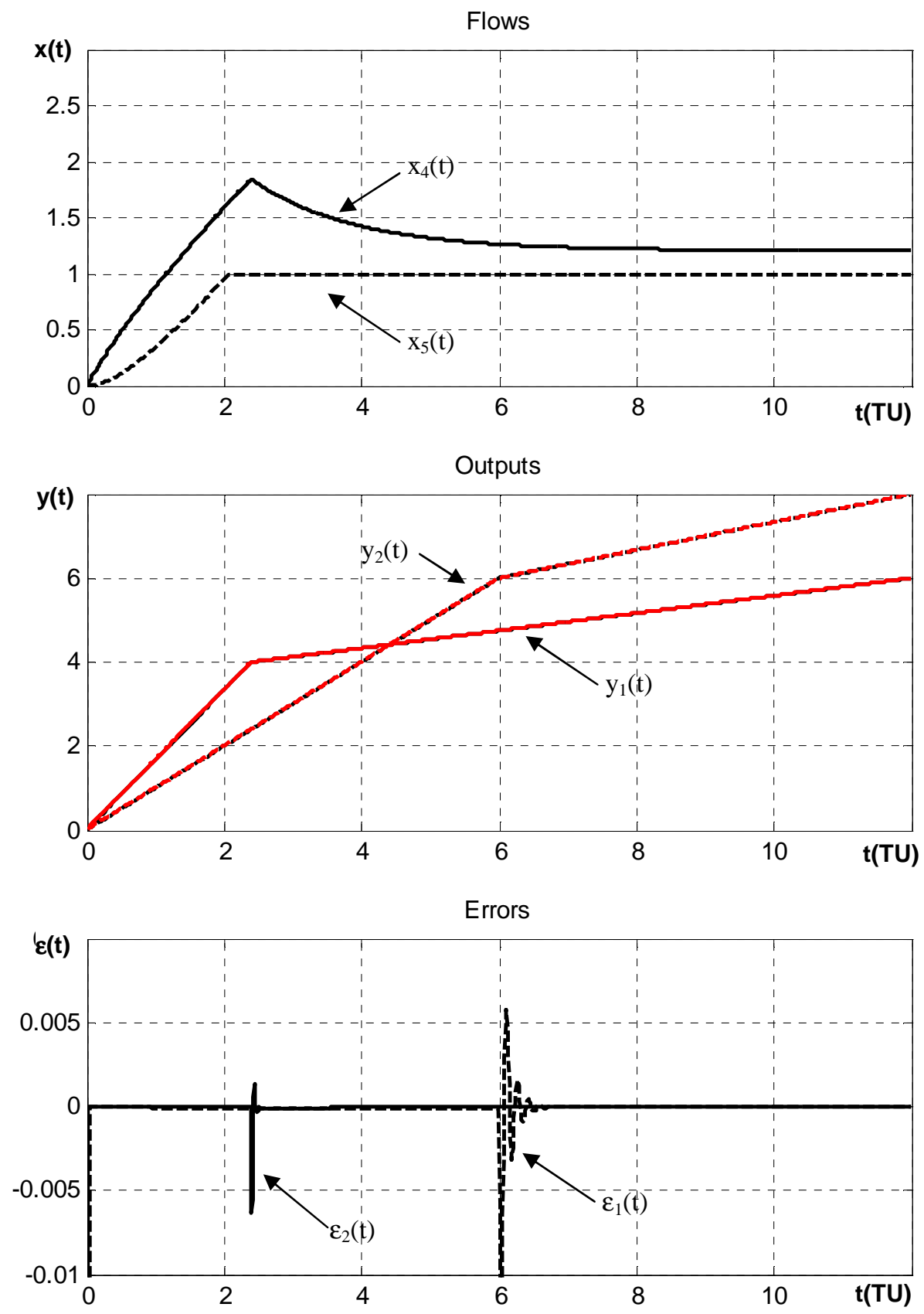

Figure 12: Control design of system C

(full line: first input, first output, dashed line: second input, second output)

[Insert figure 12 here]

Figure 14 illustrates the case of a non admissible output trajectory. The desired output signals are defined as previously and represented in figure 14 middle (in grey). The incidence relationships of the contPN have been modified according to figure 13 (system C'): 


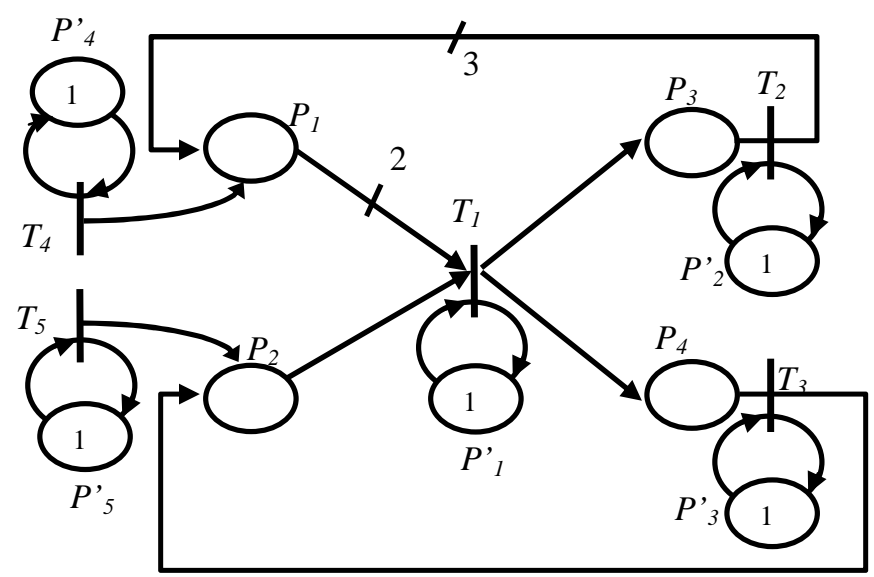

Figure 13: System C'

[Insert figure 13 here]

With system C', the marking of place $P_{1}$ increases more quickly than with system $\mathrm{C}$. In particular, in case $x_{\max 4}=x_{\max 5}=0$, system $\mathrm{C}^{\prime}$ is tokens producer but $\mathrm{C}$ is tokens consumer. After $t=4 \mathrm{TU}$, the number of tokens in the subset of places corresponding to $y_{1}$ increases more quickly than the desired output $y^{\text {des }}{ }_{1}$ and the controller fails. 

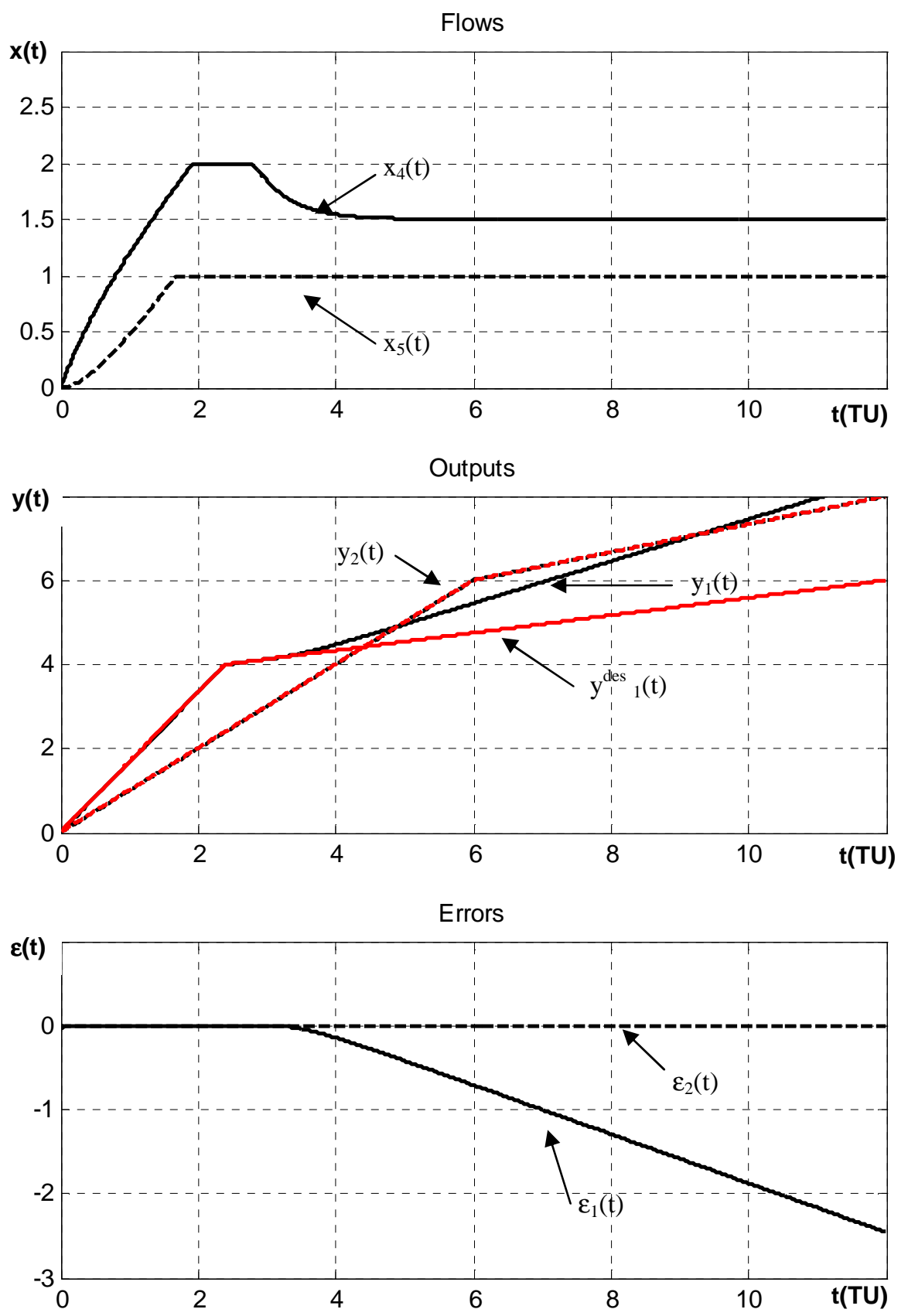

Figure 14: Control design of system C'

(full line: $x_{1}(t), u_{1}(t)$ and $\varepsilon_{1}(t)$, dashed line: $x_{2}(t), u_{2}(t)$ and $\varepsilon_{2}(t)$ ) [Insert figure 14 here]

\subsection{Control design for a hybrid system}

At last, let us consider the hybrid PN model of the two-tank system A (figure 1) given as a MIMO non linear state space representation with controllable transitions $\boldsymbol{T}_{\boldsymbol{C}}=\left\{T_{1}, T_{4}\right\}$ and outputs $y_{1}(t)=m_{1}(t)$ and $y_{2}(t)=m_{2}(t)$. The controller is obtained according to an adaptation of the gradient based algorithm to non linear behaviours $N=10$. As a consequence, the discrete part of the hybrid model becomes useless (figure 2). The desired trajectories 
correspond to a periodical level for tank 1 and a constant level for tank 2 given by equation (45):

$$
\left\{\begin{array}{l}
y_{1}^{\text {des }}(t)=\frac{1}{10} \cdot \sin \left(\frac{6 \pi}{1000} \cdot t\right)+0.5 \\
y_{2}^{\text {des }}(t)=0.4
\end{array}\right.
$$

Simulation results for GBC are given in figure 15 (system outputs are in full line, and desired trajectories are in dotted line) and can be compared with the results obtained with the discrete control design (figure 16).
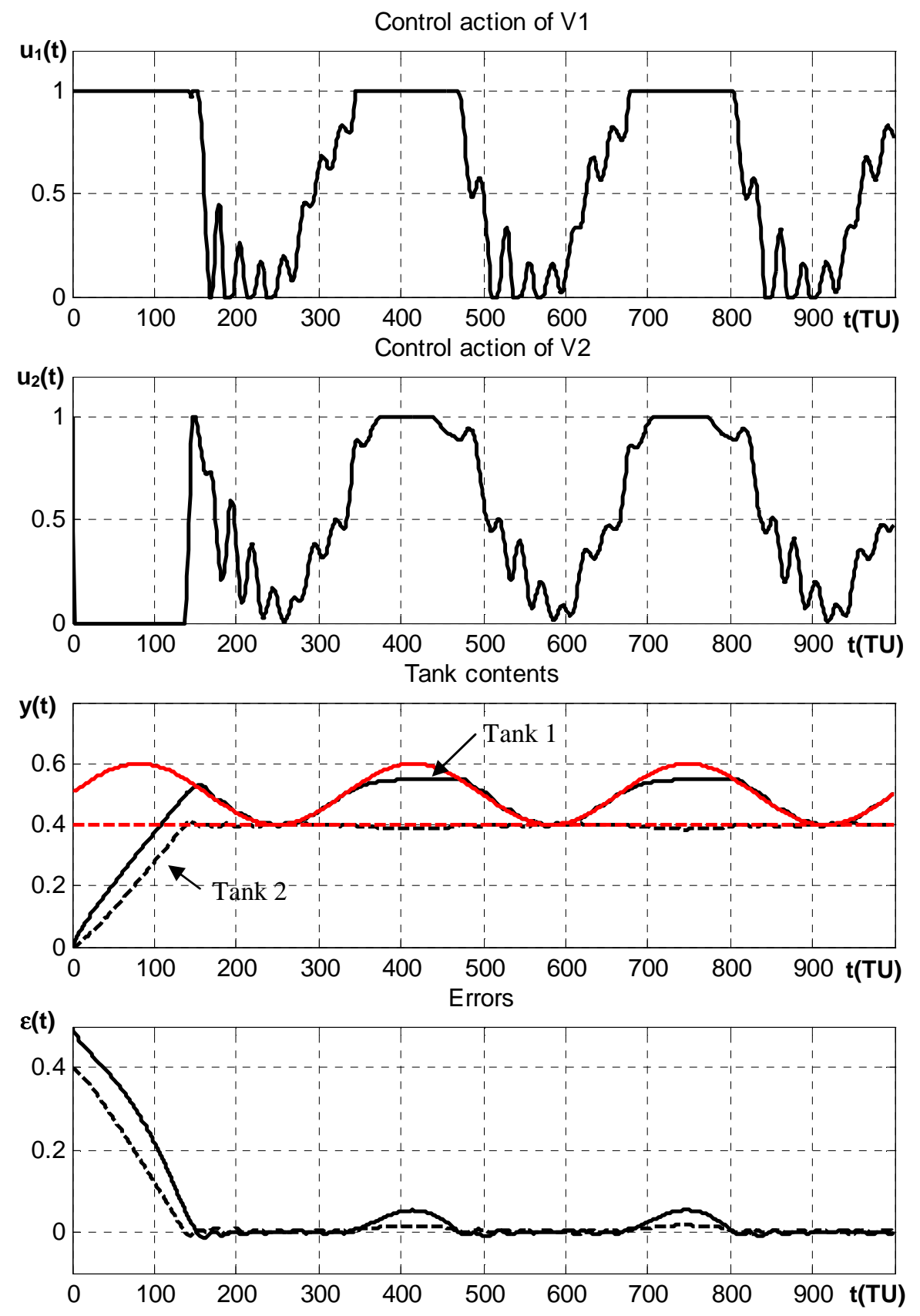

Figure 15: GBC for two-tank system 
(full line: input for $V_{1}$, output $y_{1}$, dashed line: input for $V_{2}$, output $y_{2}$ )

[Insert figure 15 here]
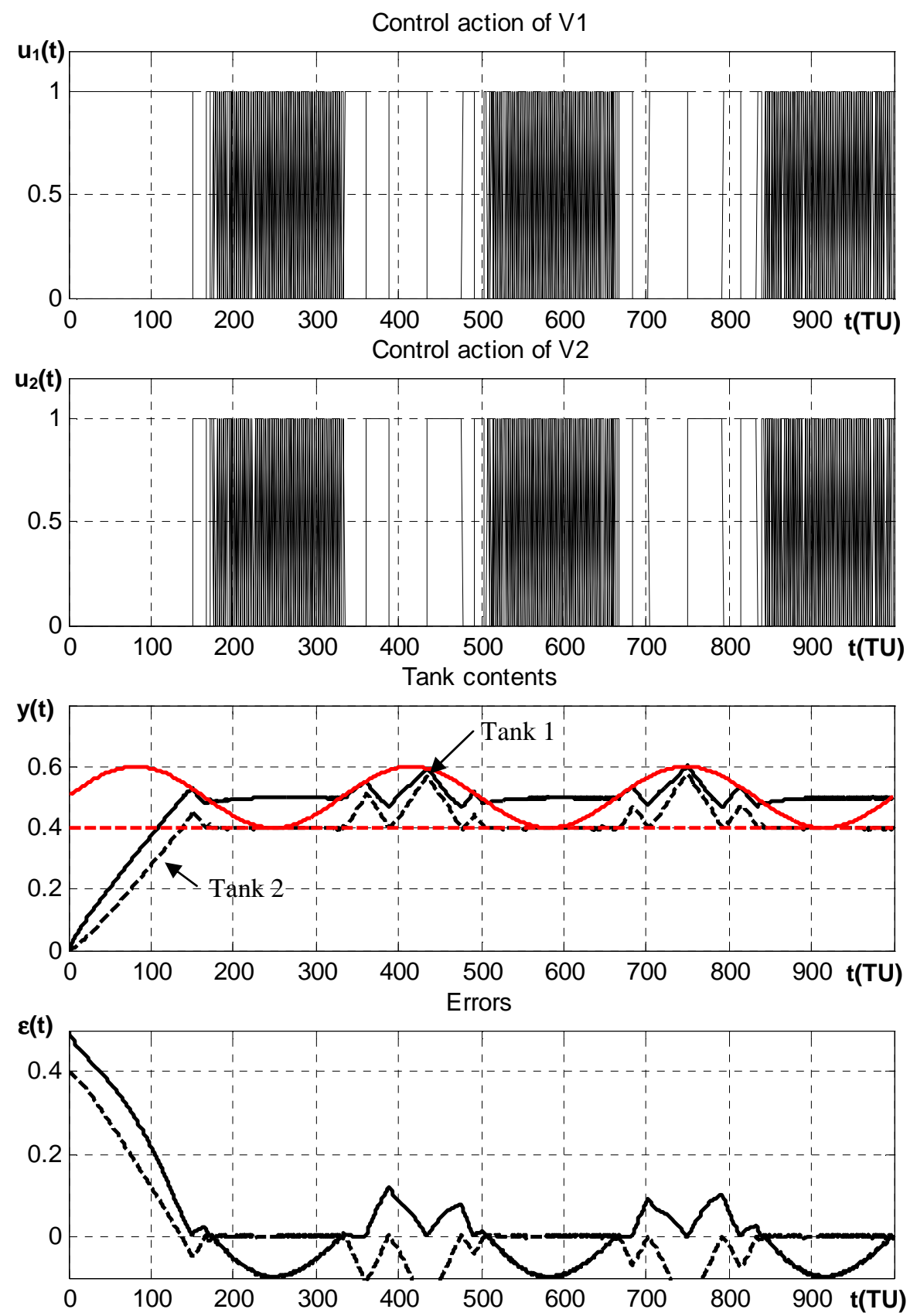

Figure 16: Discrete controller for two-tank system

(full line: input for $V_{1}$, output $y_{1}$, dashed line: input for $V_{2}$, output $y_{2}$ )

[Insert figure 16 here]

Due to the opposite position of valves $V_{1}$ and $V_{2}$, the discrete controller is not suitable to track the reference trajectories. On the contrary, with GBC, the desired level in tank 2 is reached 
and the reference trajectory in tank 1 is almost everywhere tracked after some transient behaviours. But one can also notice that, due to system specifications, level $0.6 \mathrm{~m}$ cannot be reached in tank 1 when level in tank 2 is $0.4 \mathrm{~m}$. At last, because of immediate causality relationships from $T_{1}$ to $P_{1}$ and from $T_{4}$ to $P_{2}$, GBC behaves like a proportional controller (i.e. the input - output sensitivity matrix tends to a diagonal one). Let us notice that PI, on/off and MPC controllers are not suitable for system A: PIC behaves at best like GBC but gains must be computed for each desired level, MPC is not easy to implement due to computational complexity (system A is non linear) and on/off controller behaves like the discrete one.

\section{Conclusions}

Control design has been proposed for contPN with controllable and uncontrollable transitions. The proposed controllers are based on the evaluation of sensitivity functions. For this purpose, the structural sensitivity of PN models has been first investigated. Places to be observed and transitions to be controlled are obtained as a consequence. Then, an explicit characterisation of the input-output sensitivity functions has been proposed for contPN. GBC have been designed as a consequence. Such controllers calculate the gradient of the outputs wrt the input variations in order to adapt the control actions of the controllable transitions according to desired trajectories in the output space. An application of this algorithm for HDS has been also presented.

In our opinion, the method is not only suitable for trajectory tracking but also for complex behaviours learning. We will further investigate the combination of Petri nets and adaptation algorithm in order to design learning Petri nets. These perspectives include not only the continuous Petri nets but also the autonomous and timed Petri nets.

\section{Notes on contributors}

Dimitri Lefebvre graduated from the Ecole Centrale of Lille (France) in 1992. He received his $\mathrm{PhD}$ in Automatic Control and Computer Science from University of Sciences and Technologies, Lille in 1994, and his HAB from University of Franche Comté, Belfort, France in 2000. Since 2001, he has been a Professor at Institute of Technology and Faculty of Sciences, University Le Havre, France. He is with the Research Group on Electrical Engineering and Automatic Control (GREAH) and from 2007 to 2012 he was the head of the group. His current research interests include Petri nets, learning processes, adaptive control, fault detection and diagnosis and its applications to electrical engineering. 
Edouard Leclercq received the B.S. degree in physic and mathematics from Paris Educational District in 1987 the M.S. in Electronic from University of Rouen (France) in 1994 and the Ph.D. degree in Automatic from University of Le Havre (France) in 1999. Since 1999 he is a Lecturer with the department of Electronic, Electro-technology and Automatic at the Faculty of Sciences and Technology of Le Havre (France). Between 1995 and 1999 he has been with the L.A.CO.S. (Laboratoire d'Analyse et de COmmande des Systèmes) working on Vision Machine for Movement Detection and Tracking. Since 1999 he has been with the Research Group on Electrical Engineering and Automatic Control (GREAH). His current research interests include modelling, control and fault detection using dynamical neural network. The principal applications are electrotechnical processes such as motors and wind generators.

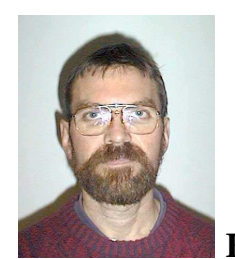

Fabrice Druaux received the B.S. degree in physic and mathematics in 1976 the M.S. in physic in 1981 and the Ph.D. degree in physic from University of Rouen (France) in 1986. Since 1988 he is a Lecturer with the department of Electronic, Electro-technology and Automatic at the Faculty of Sciences and Technology of Le Havre (France). Between 1988 and 1999 he has been with the L.A.CO.S. (Laboratoire d'Analyse et de COmmande des Systèmes) working on dynamical neural network for pattern recognition and classification. Since 1999 he has been with the Research Group on Electrical Engineering and Automatic Control (GREAH). His current research interests include modelling, control and fault detection using dynamical neural network. The principal applications are electro-technical processes such as motors and wind generators.

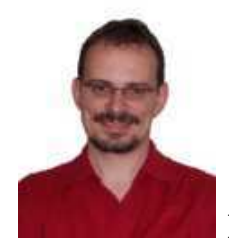

Philippe Thomas received his Ph.D. from the University Henri Poincaré Nancy 1 in 1997. He spent five years with the Systèmes et Transports Laboratory at the Technical University of Belfort-Montbéliard, where he studied model-based diagnosis using neural networks. He is currently an Associate Professor and Researcher at the Centre de Recherche en Automatique de Nancy (CRAN-UMR 7039), which is affiliated with the CNRS and Nancy University. His research interests center on the use of neural networks to perform simulation models for scheduling.

\section{References}

Apaydin-Özkan H., Julvez J., Mahulea C., Silva M., 2011, Approaching minimum time control of timed continuous Petri nets, Nonlinear Analysis: Hybrid Systems, vol. 5, pp. 136 148.

Balduzzi F., Giua A., Menga G., 2000, First-order hybrid Petri nets: a model for optimization and control, IEEE Trans. On Robotics and Automation, vol. 16, no. 4, pp. 382 -399.

Cassandras C.G., 1993, Discrete event systems: modeling and performances analysis, Aksen Ass. Inc. Pub.

Chen K.Y., 2012, Cell controller design for RFID based flexible manufacturing systems, International Journal of Computer Integrated Manufacturing, vol. 25, no. 1, pp. 35 -50. 
Chen Y., Li Z., 2012, On structural minimality of optimal supervisors for flexible manufacturing systems, Automatica, vol. 48, pp. $2647-2656$.

David R., Alla A., 2004, Continuous and hybrid Petri nets, Springer, Berlin.

Giua, A., DiCesare, F., 1994, Petri net structural analysis for supervisory control., IEEE Trans. on Robotics and Automation, Vol. 10, No. 2, pages 185-195.

Giua, A., Mahulea, C., Recalde, L., Seatzu, C., Silva, M., 2006, Optimal control of continuous Petri nets via model predictive control. In Proc. IEEE-WODES, Ann Arbor, Michigan.

Hagan M.T., Demuth H., Beale M., 1995, Neural network design, PWS publishing company, Boston, U.S.A..

Jimenez, E., Júlvez, J., Recalde, L., Silva, M., 2005, On controllability of timed continuous Petri nets systems: the join free case. Proc. IEEE-CDC, 7645 - 7650, Seville, Spain.

Júlvez J., Boel R.K., 2010, A Continuous Petri Net Approach for Model Predictive Control of Traffic Systems, Trans. IEEE-SMCA, vol. 40, no. 4 pp. 686-697.

Kara, R., Ahmane, M., Loiseau, J.J., Djennoune, S., 2009, Constrained regulation of continuous Petri nets, Nonlinear Analysis: Hybrid Systems, 3(4), 738-748.

Lee, J., Jeong I.J., 2011, A heuristic algorithm to minimise the control places of a Petri net for the control of shared machines, International Journal of Production Research, vol. 49, no. 6, pp. $1713-1730$.

Lefebvre D., 1999, Feedback control designs of manufacturing systems modelled by continuous Petri nets, International Journal of Systems Science, vol. 30, no. 6, pp. 591-600.

Lefebvre D., 2011, About the stochastic and continuous Petri nets equivalence in long run, Non-Linear Analysis, Hybrid Systems (NAHS), vol.5, pp. 394-406.

Lefebvre D., 2012, Approximation of the asymptotic mean marking of SPNs with contPNs, Non Linear Analysis: Hybrid Systems (NAHS), Vol. 6, pp. 972-987, 2012.

Lefebvre D, Delherm C., 2003, Structural sensitivity for the conflicts analysis in Petri nets, IEEE - SMC03, 7-10 october, Washington, USA, CD - ROM.

Lefebvre D., Delherm C., 2007, Fault detection and isolation of discrete event systems with Petri net models, Trans. IEEE - TASE, Vol. 4, no. 1, pp. $114-118$.

Lefebvre D. Leclercq E., 2012, Piecewise constant timed continuous PNs for the steady state estimation of stochastic PNs, Discrete Event Dynamic Systems: theory and applications, vol. 22, no. 2, pp. 179-196, 2012

Lefebvre D, Leclercq E., Druaux F., Thomas P., 2003, Source and sink transitions controllers for continuous Petri nets: a gradient - based approach, IFAC - ADHS03, 16-18 june, Saint Malo, pp. 229 - 234, France. 
Mahulea C, Ramirez-Trevino A., Recalde L., Silva M., 2008, Steady state control reference and token conservation laws in continuous Petri net systems, IEEE - TASE, vol. 5, no. 2, pp. $307-320$.

Mahulea, C., Giua, A., Recalde, L., Seatzu, C., Silva, M., 2008b, Optimal model predictive control of timed continuous Petri Nets, IEEE Transactions on Automatic Control, 53(7), pp. 1731-1735.

Ross-Leon, R., Ramirez-Trevino, A., Morales, J.A., 2012, Timed continuous Petri nets based control for metabolome under Michaelis-Menten kinetics. World Automation Congress WAC'12, Puerto Vallarta, Mexico.

Silva, M., Recalde, L., 2002, Petri nets and integrality relaxations: a view of continuous Petri nets. Trans. IEEE - SMC, part C, 32(4), 314-326.

Silva M., Recalde L. 2004, On fluidification of Petri Nets: from discrete to hybrid and continuous models, Annual Reviews in Control, vol. 28, pp. 253-266.

Thomas P., 1997, Contribution à l'identification de systèmes non linéaires par réseaux de neurones, Thèse de Doctorat, Université de Nancy.

Tolba C., Lefebvre D., Thomas P., El Moudni A., Continuous and timed Petri nets for the macroscopic and microscopic traffic flow modelling, Simulation Modelling Practice and Theory, vol. 13/5 pp. 407-436, 2005

Tuncel G., 2012, An integrated modeling approach for shop-floor scheduling and control problem of flexible manufacturing systems, Int. J. Adv. Manuf. Technol., vol. 59, pp. 1127 1142 .

Van der Smagt P.P., 1994, Minimisation methods for training feedforward neural networks, Neural Networks, vol. 7, no. 1, pp. 1-11.

Vazquez, C.R., Ramirez, A., Recalde, L., Silva, M. 2008, On controllability of timed continuous Petri nets. In HSCC 2008, M. Egerstedt and B. Misha (Eds.), 528 - 541, Springer Verlag, Berlin.

Vazquez, C.R., Silva, M., 2009, Piecewise-linear constrained control for timed continuous Petri nets. Proc. CDC-CCC, 5714 - 5720, Shangai.

Vazquez R., Silva M., 2012, Stochastic continuous Petri nets: an approximation of Markovian net models, IEEE Trans. Syst. Man Cybern. Part A 42 (3) pp. 641-653.

Wang L., Mahulea C., Júlvez J., Silva M., 2011, Decentralized control of large scale systems modeled with continuous marked graphs, 18th IFAC World Congress, Milano, Italia.

Wang L., Mahulea C., Júlvez J., Silva M., 2013, Minimum-time decentralized control of Choice-Free continuous Petri nets, Nonlinear Analysis: Hybrid Systems vol. 7 pp. 39-53. 
Widrow B., Lehr M.A., 1990, 30 years of adaptative neural networks: Perceptron, Madaline, and backpropagation, IEEE Proceedings, vol. 78, no. 9, pp. 1415-1442.

Zaytoon J., (editor), 1998, Hybrid dynamical systems, APII - JESA, vol 32, n 9-10.

Zhang W.J., van Luttervelt C.A., 2011, Toward a resilient manufacturing system, CIRP Annals - Manufacturing Technology, Vol. 60, pp 469 - 472.

Zhang X., Lu Q., Wu T., 2011, Petri net based applications for supply chain management: an overview, International Journal of Production Research, vol. 49, no. 13, pp. 3939 -3961.

Zhou, M.C., Li, Z.W., 2010, Special issue on "Petri nets for system control and automation", Asian Journal of Control, 12(3). 


\section{Figure captions}

Figure 1: Two-tank system (system A)

Figure 2: Hybrid PN of the two-tank system

Figure 3: Propagation of the perturbation near a given transition or place

Figure 4: contPN model of a manufacturing process (system B)

Figure 5: contPN model of system B'

Figure 6: Closed loop process (system C)

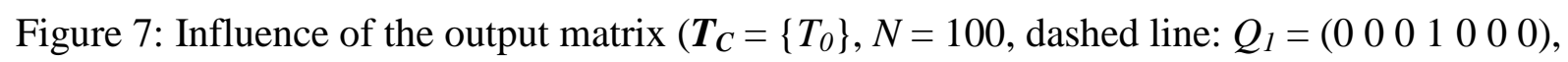

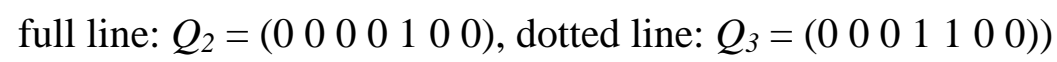

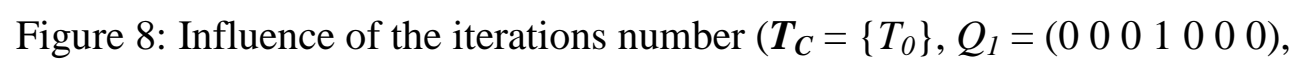

full line: $N=100$, dashed line: $N=10$, dotted line: $N=2$ )

Figure 9: Control performances comparison:

Outputs and desired output (in bold) for GBC, PIC, on/off controller and MPC.

Figure 10: Control performance comparison: Flows for GBC, PIC, on/off controller and MPC.

Figure 11: Comparison between GBC (dotted line) and MPC (dashed line) for $Q_{1}, Q_{2}$ and $Q_{3}$.

Figure 12: Control design of system $\mathrm{C}$

(full line: first input, first output, dashed line: second input, second output)

Figure 13: System C'

Figure 14: Control design of system C'

(full line: $x_{1}(t), u_{1}(t)$ and $\varepsilon_{l}(t)$, dashed line: $x_{2}(t), u_{2}(t)$ and $\varepsilon_{2}(t)$ )

Figure 15: GBC for two-tank system

(full line: input for $V_{1}$, output $y_{1}$, dashed line: input for $V_{2}$, output $y_{2}$ )

Figure 16: Discrete controller for two-tank system

(full line: input for $V_{1}$, output $y_{1}$, dashed line: input for $V_{2}$, output $y_{2}$ ) 